\title{
Lyapunov Exponents and Temperature Transitions in a Warming Australia
}

\author{
STEPHEN GILMORE \\ Diamantina Institute, University of Queensland, Brisbane, Australia
}

(Manuscript received 12 January 2018, in final form 24 February 2019)

\begin{abstract}
Multiple potential tipping points in the Earth system that involve alternative states have been identified that are susceptible to anthropogenic forcing. Past events-from millions of years ago to within the last century - have manifest as abrupt changes in climatic indicators such as the temperature record. Recent unprecedented heat waves in Australia, their associated devastation, and the considerations above provide motivation to ask whether the Australian daily maximum temperature record has been subject to such abrupt changes. Using a new diagnostic tool-the Lyapunov plot-here it is shown that multiple temperature transitions have occurred with respect to the maximum daily temperature record in widely separated locations in Australia over the last 150 years. All maximum Lyapunov exponents are positive in sign, indicating that the transitions are chaos-to-chaos transitions, and that the different climate modes identified are likely to be manifestations of distinct chaotic attractors. Many of these events occur simultaneously with transitions or extremes in the major natural cycles affecting Australia's climate, but this observation is not universal. It is known that chaos-to-chaos transitions can result in changes in the value(s) of the state variable(s) that can range from subtle to severe. Although the identified transitions are not catastrophic, this observation does not rule out the possibility of severe, unprecedented, and discontinuous increases in average daily maximum temperatures occurring in Australia at any time within the next few decades.
\end{abstract}

\section{Introduction}

Maximum daily temperature is an important variable with respect to anthropogenic climate change in Australia. Extreme temperatures have profound effects on, for example, ecology and human health, and can lead to urban electrical grid failure (Perkins-Kirkpatrick et al. 2016). Increased mortality will occur with increases in both mean daily temperatures and the number of days where the maximum is above a given threshold (Huang et al. 2012). Increased mean daily maximum temperatures, with their associated extremes (Lewis et al. 2017), are likely to increase the frequency of extreme bushfires at the urban-rural interface (Sharples et al. 2016). Recent unprecedented daily maximum temperatures in Australia (Lewis et al. 2017) including, for example, the extreme 2009 heatwave in Victoria, Australia, when Melbourne reached an unprecedented maximum temperature of over $46^{\circ} \mathrm{C}$ [this event was associated with devastating bushfires that resulted in 370 deaths and insured losses of $\$ 1.3$ billion (U.S.

Corresponding author: Stephen J Gilmore, s.gilmore1@uq.edu.au dollars) (Perkins-Kirkpatrick et al. 2016)], provide motivation to ask whether there exists evidence supporting the presence of multiple states, and by implication, tipping points or abrupt transitions, in the Australian maximum daily temperature record. The issue is important: if temperature regime shifts occur, then climate forecasting and risk assessment with respect to Australian warming will need to accommodate the existence of rapid, unexpected, and significant change as a possible outcome of the system's behavior. Indeed, the need for climate policy to account for the potential economic impacts of climate-related tipping points has been discussed elsewhere (Cai et al. 2015; Lontzek et al. 2015).

Over many spatiotemporal scales, climate can be considered chaotic. Many atmospheric systems, including the barotropic, two-layer quasigeostrophic, and primitive atmospheric systems are known to follow chaotic dynamics (Ilyin and Filatov 1988; Gorelov 1996; Lions et al. 1997). But could a climatic tipping point represent a chaos-to-chaos transition? From a theoretical perspective the answer is in the affirmative: multiple distinct parameter-dependent chaotic or strange attractors in the phase space of low-dimensional nonlinear systems are 
known to exist (Lai et al. 2018; Luo et al. 2007), indicating that chaos-to-chaos transitions in physical systems of greater complexity are overwhelmingly likely. In lowdimensional models, transitions between strange attractors exhibiting topological nonequivalence (i.e., one cannot continuously deform the shape of one attractor to the second attractor) can occur with only small changes to control parameters (Lü and Chen 2002). Furthermore, it is known that some dynamical systems may exhibit more than one strange attractor simultaneously (Zheng et al. 2004); it is thus possible a trajectory can shift to a new location in phase space following a perturbation. Some chaotic attractors are nonergodic; this means that a hitherto unexplored region of the attractor can be visited if the trajectory moves to new position secondary to a perturbation.

If a dynamical system is chaotic, mathematically simple bifurcations between chaotic attractors are unlikely. In contrast, such transitions are characterized by a wide variety of more complicated mechanisms, including boundary, interior, or merging crises (Lucarini and Bódai 2017; Thompson et al. 1994). In the boundary crisis, a strange attractor will vanish when it collides with its basin boundary; it may then be replaced by a new attractor. Interior crises are characterized by an increase in the size of the strange attractor. Merging crises occur when two or more strange attractors merge to form a single attractor. Some chaotic transitions are labeled safe, while others are labeled explosive or dangerous (Thompson et al. 1994). Finally, simple metrics, such as rolling window autocorrelation or variance measures of a time series, will have no value in the prediction of chaotic transitions since small perturbations to a trajectory in phase space will grow exponentially and not decay. Conversely, some transitions from fixed points or limit cycles to chaos may exhibit the statistical features of critical slowing down (Dakos et al. 2008) as a tipping point is approached.

Now consider the temperature record. Change in the temperature record is not always smooth: such variation may behave in a step-like manner and may thus be secondary to the crossing of tipping points associated with the boundaries of alternative climate modes. For example, recent work has revealed the existence of an abrupt increase in the sea surface temperature in the midnorthern latitudes in the 1920s and again in the 1980s (Varotsos et al. 2014), and temperature records over the last 100 years in both Russia and China reveal local and spatiotemporally coupled abrupt increases in mean daily temperatures (Anisimov et al. 2013; Tong et al. 2014). If these temperature time series are manifestations of chaos, and the transitions are due to the underlying dynamical systems transitioning between different chaotic attractors, then identifying all the tipping points or alternative states of such a system-including those where the state variable change is either subtle or masked by noise-becomes a formidable challenge (Bathiany et al. 2016).

Although it may not be possible to determine the exact type of chaotic transition from a single temperature record, in the following sections a general procedure is introduced that facilitates the identification of potential chaotic Australian climate modes and their transitions, involving three steps:

(i) Clustering of the temperature record. In the analysis each year represents a point in a multidimensional vector space. Temporal association of the yearly cluster membership, if present, will aid in the identification of different maximum daily temperature regimes.

(ii) A Lyapunov plot is calculated. This is a rolling window plot of the largest Lyapunov exponent with respect to the reconstructed attractor derived from the time series under investigation (Bryant et al. 1990). Takens' theorem (Takens 1981) provides the theoretical underpinning of attractor reconstruction. It is hypothesized that different maximum temperature regimes will manifest as different Lyapunov plot patterns.

(iii) Analysis of the Lyapunov plot. It is hypothesized that transitions between Lyapunov plot patterns will be identifiable by changes in their respective spectral signatures. Spectral analysis of the Lyapunov signal is performed; this is followed by discrete and continuous time-frequency wavelet analysis.

\section{Data}

Available daily maximum temperature data were obtained for Sydney, Melbourne, Brisbane, Perth, Adelaide, Hobart, and Darwin from the Australian Bureau of Meteorology website (www.bom.gov.au). Strict quality control after 1910 implemented by the newly established Australian Bureau of Meteorology (ABM) facilitated the collection of homogeneous data. Pre-1910 data from Sydney and Melbourne are considered to be of good quality. All seven datasets were derived from unchanged locations. While all datasets were over $99 \%$ complete, the duration of the dataset varied with the city. Melbourne has the longest continuous dataset, beginning in 1856 , corresponding to 58035 daily maximum temperature values (see Table 1). Detailed historical metadata (e.g., regarding the dates of instrument changes) are publicly available at the ABM website for all seven capital city weather station 
TABLE 1. Temperature records used in the analysis. Note the low percentage of missing values. The number of windows per year in each city is identical, while the duration of that window varies as per the analysis described in section $3 \mathrm{~b}$. Note the anomalous lag in Darwin (calculated when reconstructing the associated attractor from the denoised and bandwidth-smoothed time series). This is likely to be secondary to the observed minimal variation in daily maximum temperatures at this near-equatorial location. Note the low-dimensional embedding dimensions (ED) found in all cities.

\begin{tabular}{|c|c|c|c|c|c|c|}
\hline & Data years & No. of values & Missing values (\%) & $\begin{array}{l}\text { No. of windows } \\
\text { per year }\end{array}$ & $\begin{array}{c}\text { Window length } \\
N \text { (days) }\end{array}$ & $\begin{array}{l}\text { LE calculation time } \\
\text { series lag/ED }\end{array}$ \\
\hline Sydney & 1859-2016 & 57670 & 0.0026 & 37 & 34 & $5 / 8$ \\
\hline Melbourne & $1855-2015$ & 58035 & 0.0001 & 37 & 45 & $4 / 8$ \\
\hline Perth & $1945-2016$ & 26280 & 0.0011 & 37 & 25 & $5 / 9$ \\
\hline Brisbane & 1944-2017 & 26946 & 0.0055 & 37 & 17 & $8 / 8$ \\
\hline Adelaide & $1955-2017$ & 22299 & 0.0001 & 37 & 39 & $5 / 9$ \\
\hline Hobart & 1917-2016 & 36170 & 0.0003 & 37 & 42 & $6 / 8$ \\
\hline Darwin & $1941-2016$ & 27410 & 0.0089 & 37 & 51 & $89 / 8$ \\
\hline
\end{tabular}

locations that have provided data for the analysis presented here. All data analysis described in the following sections were performed in the R v3.1.2 (R Core Team 2014) or Mathematica v11.1.1 (http://www.wolfram.com/) environments.

\section{Methodology}

\section{a. Data preprocessing}

Initially, data for 29 February (leap year data) were removed. These data were not normalized or centered to zero. Gaps in the temperature record were filled using a linear interpolation function. These data were then subject to a local-projection filter to remove noise (Kantz and Schreiber1997), utilizing an attractor reconstruction where the appropriate time delay was determined using the first minimum of the autocorrelation (Kantz and Schreiber 1997), and the appropriate embedding dimension estimated using the method of false nearest neighbors (Kennel et al. 1992). Please refer to section $3 f$ for a discussion regarding attractor reconstruction (and thus an explanation of the term "embedding dimension"). Noise here is a consequence of errors in the measurement and recording process, and intermittent sampling error due the location of the measuring device not always being representative of the region in general.

\section{b. Bandwidth smoothing: Reducing the size of the yearly dataset}

To facilitate clustering and the generation of rolling window plots with respect to the whole dataset spanning all years (see sections 3c-e below) the yearly dataset (365 values) for all years and all cities are initially reduced to a smaller number of values. This bandwidth smoothing procedure calculates the mean with respect to a succession of data windows spanning an entire year's 365 daily values. Generating these windows requires the specification of two parameters: the lag $L$ and the window length $N$.
Here, the aim is to generate 1000 data points that span 30 years of the daily temperature record. A lag $L$ of 10 days is therefore chosen, yielding 37 windows (and thus mean values) per year. A minimum of 1000 data points will permit an accurate calculation of the moments of distribution and Lyapunov exponents (Ditlevsen and Johnsen 2010), and 30 years is considered the standard duration representing climate by both the World Meteorological Organization (WMO) and the Intergovernmental Panel on Climate Change (IPCC). Consider, for example, a window of length $N=20$. With $L=10$, each year's data are thus transformed to 37 overlapping windows containing daily maximum temperatures: 1-20 January, 11-30 January, 21 January9 February, etc. Taking the mean of all values within each window then yields 37 data points.

To determine the optimal value of $N$, the variance of each of these windows is calculated, and the mean of these 37 values is then used to characterize the variance associated with that window length for that year. Minimizing this average variance with respect to a range of window lengths results in a specific window length that yields a mean daily maximum temperature value for any window that most accurately represents all these data contained in that window. To see why, consider the extreme case: if the variance was equal to zero for a window of given length, then its associated mean value is identical to all the values found in that window.

For any given year, the optimal value of $N$ is found at the local minimum of the variance versus window length plot; an example of this plot for Sydney in the year 1870 is shown in Fig. 1a. This plot shows the average value of the variance, computed, for one year, for all windows where $L=1$ and the window length $N$ ranges from 1 to 80 days. For example, note that when $N=60$ days, the average variance of all lagged windows for the entire year is 5.5.

Taking the mean value of all yearly window lengths $N_{i}$ associated with minimums in the variance yields a 

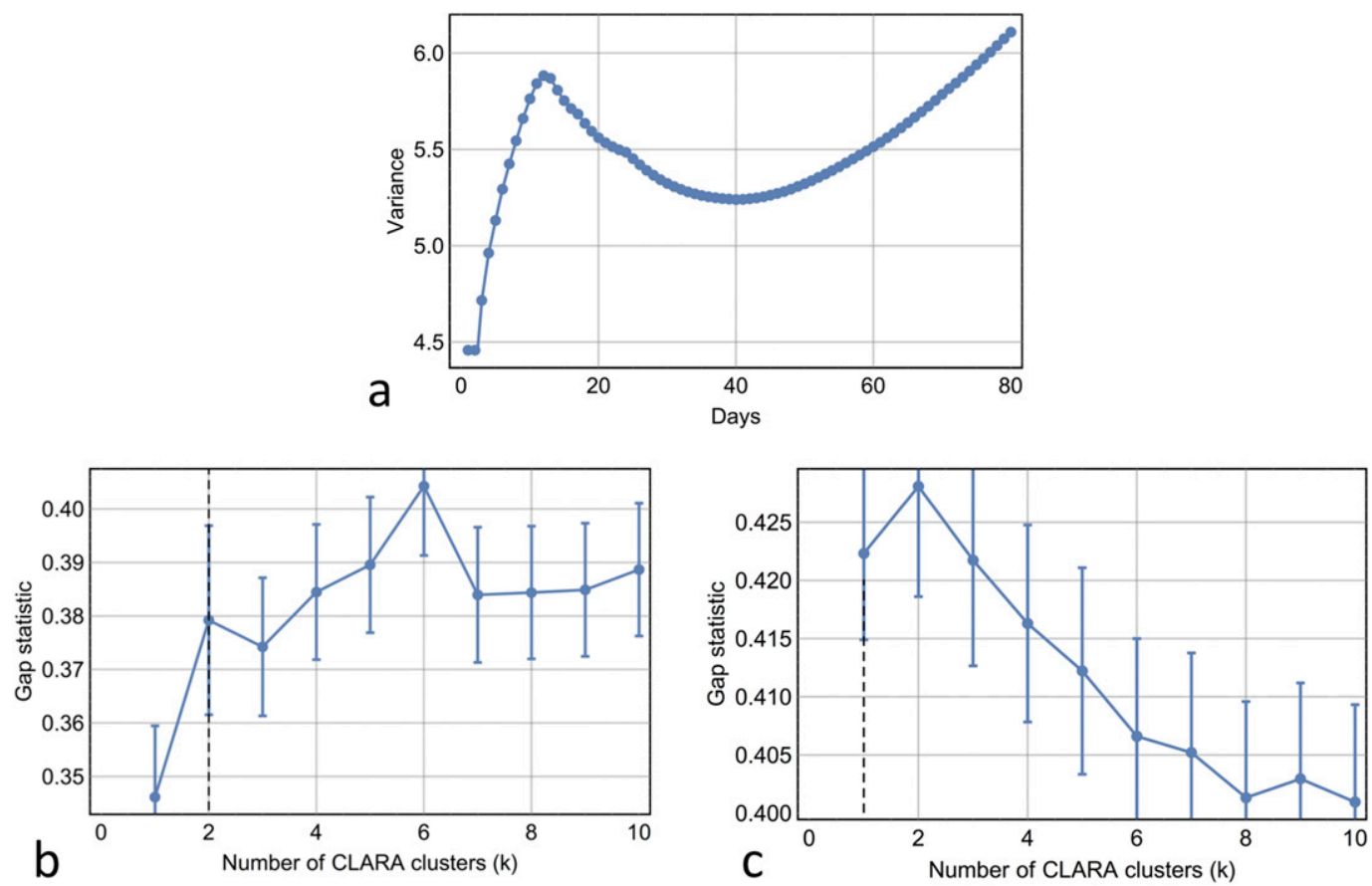

FIG. 1. (a) Variance in daily maximum temperature as a function of rolling window duration for Sydney for the year 1870. Note the local minimum at 42 days. (b),(c) Gap statistic as a function of number of CLARA generated clusters. In this example, note the optimal cluster size (given by the dashed line) is two for these Sydney maximum temperature data in (b), while appropriate random Gaussian data about the least squares fit (thus controlling for deviation and trend) in (c) reveals an optimal cluster size of only one.

value $\langle N\rangle$, which is then applied to all years (with respect to each city). Taking the mean with respect to all values in each of 37 windows thus reduces 365 values to 37 values (for each year). Each mean value then characterizes the daily maximum temperature for a given year and at a particular time of year.

Finally, note that for the purposes of comparing temperature data between years, window lengths must be the same regardless of the year, and short durations where the variance is trivially small (see Fig. 1a, where $N<7$ ) are a poor choice in characterizing a particular time of year since the mean will be derived from data associated with short-term weather systems-which will be highly variable from year to year.

\section{c. Clustering}

Since each year's data are reduced to 37 values, any year can be characterized by a point in a 37-dimensional vector space, where each dimension represents a particular time of year. If, for example, the dataset contained 100 years of data, this would result in 100 points existing within the 37-dimensional vector space.

To test the hypothesis that different regimes of maximum temperature are present in these data, all points in the vector space were initially subject to four clustering
algorithms-K-means, Partition about mediods (PAM) (Reynolds et al. 2006), Clustering Large Applications (CLARA), and Fuzzy Analysis (FANNY) (Maechler et al. 2014) - where in each case the optimal number of clusters was determined by either the gap-statistic method (Tibshirani et al. 2001) (Fig. 1b) or the elbow method (Ketchen and Shook 1996). Note that this analysis permits an optimal cluster size of one if these data do not meaningfully partition into two or more clusters. Although the number of interior points (corresponding to the number of years in the temperature record) are much less than the number of vertices of a 37-dimensionsal hypercube, identical clustering algorithms are validated in the bioinformatics community, where, for example, the analysis might cluster 50 points into two or more groups within a 10000-dimensional hypercube (Oyelade et al. 2016).

To illustrate the clustering of data, consider $K$-means clustering. Here the aim is to partition the dataset into $K$ clusters such that the sum of squares of the distances of each data point $x_{i, k}$ to the centroids $C_{k}$ of the assigned clusters

$$
\sum_{k, i}^{K, N}\left\|C_{k}-x_{i, k}\right\|^{2}
$$

is minimized. 
To exclude the possibility that any clustering is a spurious consequence of the trend in these data, control data (generated by determining the least squares linear fit with suitable Gaussian noise superimposed) were used as a control (Fig. 1c).

To validate the results with respect to the optimal number of clusters, the package nbcluster in $\mathrm{R}$ was then applied (Charrad et al. 2014). In total, these data were subjected to 241 different combinations of clustering and optimal-cluster-number determining algorithms, where the optimal cluster size is two or more. Finally, these data were partitioned specifying either 2 or 3 clusters using the four algorithms noted above, where the FANNY algorithm yields a probability of class membership, but can also perform a hard clustering. Dunn's Partition Coefficient (Dunn 1974) was then calculated with respect to the $F A N N Y$-generated clusters.

\section{d. Detrending: Statistical properties}

To investigate the statistical properties of the temperature record, instead of representing each year's data as a point in a 37-dimensional vector space, these data are used in their original chronological order, resulting in a list of values of length $37 Y$ where the duration of the dataset is $Y$ years. The first moment of distribution (the mean) was then calculated using a rolling window of length 1000 and lag 1 . In this case the rolling window methodology generates a series of overlapping windows of length 1000 and lag 1 that covers these data. Using a lag of 1 maximizes the number of data points in the rolling window plots. The mean is then calculated with respect to each window; a succession of means are then plotted at the time corresponding to the last data point of its associated window. Each value of the rolling window plot thus represents the mean of the previous 1000 data points, corresponding to 30 years, while the plot is referred to as a mean maximum temperature plot (MMT).

Detrended data were then obtained with minimal bias by subtracting the least squares linear approximation from the trended data. The second (variance), third (skewness), and fourth (kurtosis) moments of distribution with respect to the residuals were then calculated using a rolling window—as for the mean calculation above-with length 1000 and lag 1.

\section{e. Dynamical systems and Lyapunov exponents}

The concept of the Lyapunov exponent is derived from a consideration of dynamical systems theory. Dynamical systems theory deals with the evolution of physical systems in time (Strogatz 1994). The behavior of simple physical dynamical systems with a small number of variables - such as an undamped pendulum swinging in a plane-can be described accurately by solving a set of differential equations. Given a set of initial conditions, solutions to these differential equations yield the position and velocity of the pendulum as a function of time. But the solutions can be visualized as a trajectory, or orbit, in phase space, where, in the case of the pendulum, the axes are its position and velocity. In general, the trajectories of dynamical systems in phase space converge to the attractors of that system, where the latter are of three types: (i) fixed point, (ii) limit cycle, and (iii) chaotic. For a fixed point attractor, the system will come to rest; for a limit cycle attractor, the system will repeat a sequence of identical states indefinitely; and finally, for a chaotic attractor, the system will never repeat any state exactly but will remain within a bounded region of the phase space. The basin of attraction for a given attractor comprises all regions within the phase space where a trajectory, if beginning in that region, will converge to its corresponding attractor.

Perturbations of dynamical systems may be due to changes in the values of one or more parameters of the system or due to changes in the values of one or more of its dynamical variables. Slow forcing of a parameter of a chaotic system may be associated with threshold-type behavior: when the parameter reaches a critical value, an abrupt change in the shape of the system's underlying attractor may occur (Lü and Chen 2002). As noted in the introduction, when an attractor abruptly changes, a bifurcation has occurred. A bifurcation can therefore be associated with a discontinuous change in the values of one or more of the system's dynamical variables. On the other hand, an externally imposed short-duration change in the values of one or more dynamical variables of a chaotic system will not alter its underlying attractor. The system will, however, now follow a very different phase space trajectory (usually, however, within the same attractor) in comparison with the trajectory that would have ensued without the perturbation. This latter phenomenon is known as sensitivity to initial conditions because the magnitude of the initial perturbation can be arbitrarily small.

Lyapunov exponents can be thought of as a measure of the stability of a dynamical system (Williams 1997). For a stable fixed point or a stable periodic orbit, the values of the Lyapunov exponents are negative, indicating that following a short-duration perturbation to the value of a dynamic variable, a perturbed trajectory converges toward the trajectory that would have ensued without the perturbation. In contrast, chaotic orbits are associated with positive Lyapunov exponents, indicating that initially closely separated orbits diverge. The magnitude of a positive Lyapunov exponent quantifies this initial rate of divergence (Williams 1997); its value thus provides a measure of the degree of instability of a 
chaotic system. However, despite this instability, initially closely separated trajectories will not continue to diverge for large times because they are still bound by their common attractor.

For small distances and times, the projection of the difference vector between initially close trajectories onto the $n$ orthogonal axes of the phase space will form an ellipsoid in the $n$-dimensional space. In general, the rate of asymptotic expansion or contraction of this ellipsoid with respect to its $n$ dimensions corresponds to the Lyapunov spectrum, where the $n$ Lyapunov exponents are defined as

$$
\lambda_{n}=\lim _{t \rightarrow \infty} \frac{1}{t} \log \frac{L_{i}(t)}{r(0)} .
$$

Here $r(0)$ is the radius of the initial volume element and $L_{i}(t)$ the length of the $i$ th principal axis at time $t$. The longest axis of the ellipsoid corresponds to the most unstable direction of the flow, hence its rate of asymptotic expansion corresponds to the largest Lyapunov exponent. For chaotic attractors, at least one value within the Lyapunov spectrum must be positive, indicating that initially close trajectories will diverge at an exponential rate. A global largest Lyapunov exponent, which will always be positive, is an invariant with respect to a given chaotic attractor.

\section{f. Lyapunov exponent calculations}

Lyapunov exponents can be accurately calculated if the differential equations governing a dynamical system are known. However, in the case where a single time series is the only information available, it is still possible to reliably estimate the maximal Lyapunov exponent of the underlying dynamical system's attractor by exploiting Takens' theorem and first performing an attractor reconstruction (Takens 1981). An attractor reconstruction generates a matrix $\mathbf{R}$ of values

$$
\mathbf{R}_{E, N}=\{y(t), y(t+\tau), y(t+2 \tau), \ldots, y[t+(E-1) \tau]\}
$$

using lagged coordinates of a single time series $y(t)$ with lag $\tau$. With dimensions $E \times N$, $\mathbf{R}$ thus defines a reconstructed attractor consisting of $N$ points in an $E$-dimensional phase space, where $E$ is the embedding dimension. Takens' theorem shows that a reconstruction $\mathbf{R}$ with embedding dimension $E$ derived from a single time series $y(t)$ corresponds to a topologically equivalent attractor in comparison with the real attractor (Takens 1981). Topologic equivalence between attractors implies equidimensionality and is another way of stating that a continuous deformation can transform the shape of one attractor to the other. The proof of Takens' theorem involves advanced concepts in abstract differential topology (Huke 1993; Takens 1981) and thus its description is beyond the scope of this paper.

Reconstructing an attractor is performed by first calculating an appropriate lag $\tau$ and embedding dimension $E$ (Constantine and Percival 2014). The lag is defined here as the first minimum of the autocorrelation versus lag plot (Kantz and Schreiber 1997), while the embedding dimension is calculated here utilizing the method of false nearest neighbors (Kennel et al. 1992). Consider an example of attractor reconstruction: a time series $y(t)$ composed of 1000 values given by an associated $\operatorname{lag} \tau=5$ and embedding dimension $E=8$ will yield a matrix $\mathbf{R}$ of dimensions $8 \times 965$. The values of this matrix thus define an attractor characterized by 965 successive points in an 8-dimensional phase space.

Although 300 time series measurements have been used perform attractor reconstruction and calculate Lyapunov exponents in a chaotic system (Dechert and Gencay 1992), it is considered advantageous to use a larger number of data points; hence the window applied here comprises 1000 time series values. The noisereduction procedure outlined in section $3 \mathrm{a}$ above is applied to these original temperature data since Lyapunov exponent values may be sensitive to the presence of noise (Dechert and Gencay 1992).

Following Bryant et al. (1990) and Abarbanel et al. (1993) the global maximal Lyapunov exponents are calculated by averaging over a series of regularly spaced reference points with respect to a reconstructed attractor derived from the time series window under investigation (Constantine and Percival 2014). Each Lyapunov spectra at these reference points on the reconstructed attractor are given by the eigenvalues of the Oseledec matrix - a matrix product of successive local Jacobians and their respective transposes. For example, an eight-dimensional phase space results in an $8 \times 8$ size Jacobian of partial derivatives [please see Bryant et al. (1990) and Abarbanel et al. (1993) for technical details]. With respect to the attractor flow, these eigenvalues characterize the rate of divergence (if positive) or convergence (if negative) of the orthogonal projection of trajectories associated with arbitrarily close initial points. The global Lyapunov spectrum simply averages the Lyapunov spectra at all reference points; the global maximum (the maximum Lyapunov exponent) is then the largest value with respect to the averaged spectra.

Although spurious positive Lyapunov exponents may occur in association with random time series (Tanaka et al. 1996), in contrast to Lyapunov exponents derived from chaotic dynamics, the values of spurious positive Lyapunov exponents are an artifact of the sampling size 
of the time series analyzed (Fernandez-Rodriguez et al. 2005). In general, initially close trajectories will diverge linearly in the phase space of attractors derived from random time series; hence the associated Lyapunov exponents will be of magnitude zero. Importantly, in contrast to what is expected by analyzing a random time series, in all these time series analyzed here, the fraction of false nearest neighbors dropped to zero with a lowdimensional embedding (see Table 1). The application of this false nearest-neighbor test (Kennel et al. 1992) thus reveals that all these temperature time series fail this test for randomness.

In previous work, Lyapunov exponents have been calculated with respect to simple climate models in order to determine whether the system is chaotic (Mihailovic et al. 2014). Changes in the (still chaotic) dynamical regime due to parameter variation manifest as changes in the (still) positive value of the largest global Lyapunov exponent. Phase transitions occurring within the chaotic regime are manifest as discontinuities in the values of the positive maximum Lyapunov exponents (Kluiving et al. 1992).

\section{g. The Lyapunov plot}

It is possible to track the temporal variation in the value of the largest global Lyapunov exponent with respect to the detrended time series data using the rolling window methodology. The plot, which is analogous to the moments of distribution plots described above, with a rolling window length of 1000 and lag 1 , is called a Lyapunov plot. As for the moments of distribution rolling window plots, choosing a lag of 1 maximizes the number of points in the plot.

\section{h. Lyapunov plot power spectra}

To characterize Lyapunov plot patterns, the power spectrum was calculated with respect to specific Lyapunov plot patterns, generating power versus frequency curves. To determine which frequency (if any) best distinguishes between different Lyapunov plot patterns the power over a range of frequencies was investigated with respect to specific Lyapunov plot patterns. Given the existence of an optimal frequency, rolling window plots of the power at this optimal frequency with respect to their associated Lyapunov plots-spectral power plots-were then generated using a window length of 500 and lag 1.

\section{$i$. Discrete and continuous time-frequency wavelet analysis}

Seven transitions in the Lyapunov signal identified by the procedures above are subject to discrete (Daubechies 4) and continuous (second derivative Gaussian) time-frequency wavelet analysis (Torrence and Compo 1998), utilizing a segment of the Lyapunov plot of length 1000 , where the center of the segment is the transition time. To reduce the effects of boundary artifacts, the Lyapunov signal is padded with 100 zeros at either end.

\section{The Lorenz system}

To gain preliminary insight into the nature of the patterns and transitions associated with the Lyapunov plot, consider the Lorenz system. Although the Lorenz system is introduced here as a prototypical chaotic system, it was introduced by a meteorologist (Lorenz 1963) and exhibits many qualitative similarities with the largescale atmosphere, including regime structure (Palmer 1993). Figure 2a shows a typical Lorenz chaotic trajectory from $t=0$ to $t=270$ in phase space for $\sigma=3, \rho=$ 26.5 , and $\beta=1$. At $t=270$ the trajectory is continued with the coefficient $\sigma$ of $y(t)$ changed from the value 3 to 8.1. The resultant trajectory from $t=270$ to $t=540$ is shown in Fig. $2 b$; this reveals the existence of a new chaotic attractor. In this example a new chaotic attractor is generated by a sudden and large change to the value of the parameter $\sigma$. Although this scenario does not correspond to a physically realistic situation, it reproduces the sudden appearance of a new or altered chaotic attractor (which, as discussed in the introduction, can occur in other low-dimensional models with the slow forcing of a control parameter).

An example time series of $y(t)$ before, at and after the transition is shown in Fig. 2c. The Lyapunov plotcalculated using the methods described in sections $3 \mathrm{e}, 3 \mathrm{f}$, and $3 g$ above, and generated from the scalar values $y(0)$ to $y(540)$ using a rolling window of 122 time units (1220 increments) with lag 1 -is shown in Fig. 2d [similar changes are seen using the scalar variables $x(t)$ or $z(t)]$. The first dashed line represents the transition time and the second dashed line gives the first time at which the Lyapunov exponents are calculated using reconstructed coordinates solely derived from scalar values $y(t)$ of the second attractor. It is important to note that while there are two transitions in the Lyapunov signal, there is only point in time when the system switches to the new attractor. This latter observation will be considered in relation to the Lyapunov plots generated from the temperature data in the discussion. Note that although an immediate transition to a new attractor has occurred (Fig. 2b), the extreme values of the state variable $y(t)$ have contracted but not changed catastrophically (Fig. 2c).

Note that the signal associated with either attractor is oscillatory with a period of approximately 200 time increments. Note that the Sydney Lyapunov plot (Fig. 4e) 

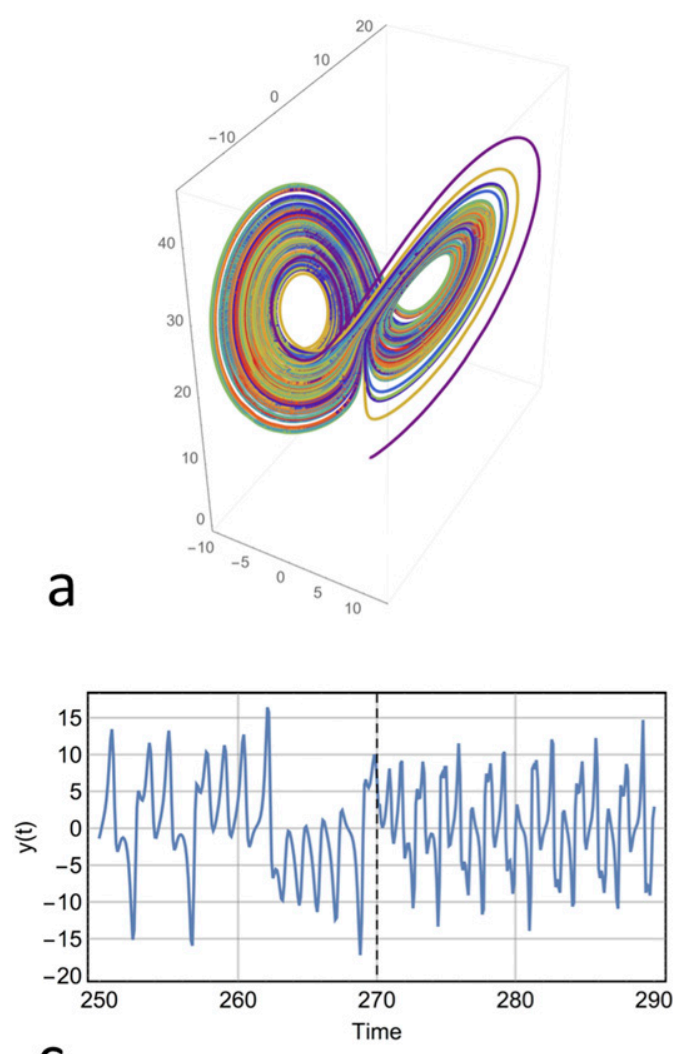

C

FIG. 2. (a) Typical Lorenz chaotic attractor mapped by following a trajectory from $t=0$ to $t=270$. (b) Following an abrupt change in the value of the parameter $\boldsymbol{\sigma}$ at $t=270$, the subsequent trajectory from $t=270$ to $t=540$ maps to a new chaotic attractor. (c) The time series $\mathbf{y}(t)$ of the first attractor from $t=250$ to $t=270$ exhibits a highly irregular oscillation, while the time series $\mathbf{y}(t)$ associated with the second attractor from $t>270$ is associated with a less irregular oscillation. (d) The associated rolling window Lyapunov plot prior to and following the transition at $t=270$. Note that both Lorenz attractors exhibit a characteristic signal, and the interval between the dashed lineswhere the Lyapunov exponents are derived from a reconstructed attractor obtained from the time series $\mathbf{y}(t)$ that spans both Lorenz attractors-exhibits a pattern where the large-amplitude signal almost vanishes and the signal becomes nonstationary.

also exhibits an oscillatory signal with a period of approximately 200 time units. This finding is not a coincidence. It is a consequence of the regular spacing between reference points along the reconstructed attractor used to calculate Lyapunov exponents, and the rolling window methodology. Since the reference points on the reconstructed attractor are 216 time steps apart, the reference points will realign when the rolling window has moved 216 places. Given that there are five reference points, and since the reconstructed attractor is only slightly less than 1000 time steps in length (note: the original time series is 1000 steps in length), four of these will be identical but only one will be new. The average of all five local Lyapunov exponents will therefore tend to be similar to its value 216 steps earlier. This may lead to an apparent periodicity in the signal.

However, there are four key features of the Lyapunov plot determined by the time series that are independent of the period, and thus allow the signals to be characterized. First, the large-scale amplitude of the signal will vary. In many cases, the periodicity will vanish and the amplitude will be markedly diminished. Second, the shape of the signal is independent of any periodicity. Third, the "jaggedness" of the signal will vary depending on the time series sequence used to construct it. And finally, the signal may be either stationary or nonstationary; in the latter, the magnitude of the Lyapunov exponents is evolving. Given these considerations, it is clear that each attractor reconstructed from a scalar time series will exhibit its own typical Lyapunov plot pattern. This is apparent in the Lyapunov plot associated with the Lorenz attractor. The most striking difference is the amplitude of the signal: the Lyapunov plot associated with the second reconstructed attractor exhibits a much larger amplitude in comparison with the first. Note that the transition time at $t=270$ is clearly discernable from 
the plot, and is associated with a section where the plot becomes nonstationary and has lost much of its large-amplitude periodicity. As noted above, in this region-between the dashed lines in Fig. 2d-the Lyapunov exponents were derived from a reconstructed attractor where the time series is obtained from both Lorenz attractors. Finally, note that although the mean of the Lyapunov plot values clearly identify different attractors (Fig. 2d), these values remain invariant to the system existing in different lobes of the same attractor.

\section{Results}

\section{a. Sydney}

The solution to the problem of determining the rolling window length $N$, for the year 1870, is shown in Fig. 1a. In this example, the optimal nontrivial duration, corresponding to the local minimum, was found to be 41.26 days. Repeating this calculation for all years, and taking the mean of all minimums yields an optimal duration, for all data, of 34.01 days, such that $N=34$ days (see Table 1 for the derived values of $N$ for all other cities).

Clustering of all 137 points in the vector space revealed a majority-rule unsupervised optimal cluster size of two. Trended control data revealed an optimum cluster size, independent of the clustering algorithm, of one. Applying 241 different combinations of clustering and optimal-cluster-number determining algorithms, where the optimal cluster size is two or more, reveals a majority-rule cluster size of two, followed by three.

Figure 3 shows the results of $F A N N Y$ hard clustering; this outcome is almost identical to the clustering patterns observed with $K$-means, PAM, and CLARA clustering. Dunn's partition coefficient (DPC) for the clustering in Fig. 3 is found to be 0.892 and 0.767 , respectively, indicating the presence of well-defined clusters. Furthermore, Fig. 3 reveals temporal aggregation of the generated yearly cluster membership; these results suggest that there was a major transition in Sydney's maximum daily temperature in the late 1910s, and a second transition in the mid-1980s (note the position of the arrows, Fig. 3). These transitions were associated with warming; the centroid coordinate values of the three clusters shown in Fig. 3 (bottom) reveal progressive increases.

Note from Fig. 4a (the MMT plot) that the trend for Sydney over the last 150 years is toward higher daily maximum temperatures. Rolling window plots for the variance, skewness, and kurtosis are shown in Figs. 4b-d, respectively. Note that the variance reaches a maximum at the cluster-identified transition in 1918, and that the kurtosis increases sharply following the cluster-identified transition in 1985.

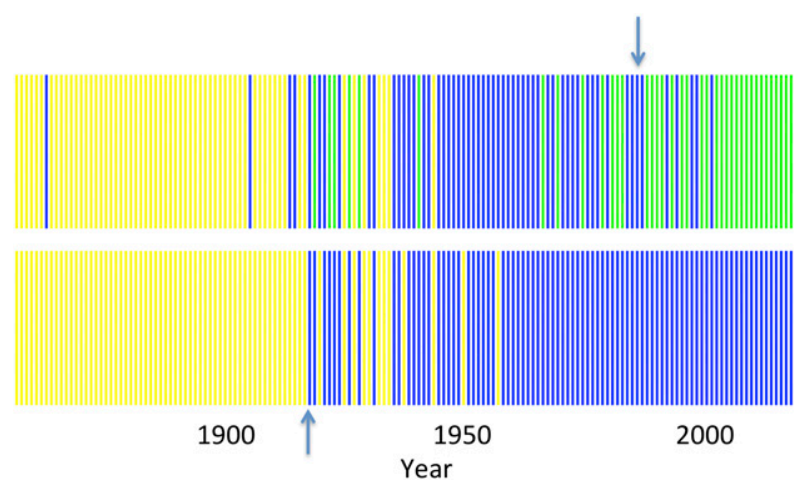

FIG. 3. (top) Three-level FANNY clustering of Sydney data for the years 1859-2016. Note the clustering membership (each vertical bar represents one year and is coded yellow, blue, or green) partitions into a well-defined temporal pattern. (bottom) Two-level FANNY clustering shows that the cluster membership partitions into a well-defined temporal pattern. The arrows at 1918 and 1985 indicate potential transition times.

Inspection of the Lyapunov plot (Fig. 4e) reveals an irregular periodic signal with varying amplitude and jaggedness. Importantly, significant changes in the amplitude and pattern of the signal occur precisely at the two transition times determined from the clustering considerations above (dashed line, Fig. 4e).

\section{b. Other Australian capital cities}

While unsupervised analysis of the temperature data for Melbourne did not reveal any significant clusters of size two or above, applying all 241 cluster-determining algorithms revealed a majority rule cluster size of two. Two-level supervised K-means, PAM, CLARA, and $F A N N Y$ clustering revealed temporal aggregation of the yearly cluster membership (see Table 1) in the 1980s (at the same time as the Sydney cluster boundary). Twolevel FANNY clustering reveals a DPC of 0.770 , indicating reasonably well separated clusters. The MMT plot for Melbourne is shown in Fig. 5a, revealing a trend of increasing temperatures. The second, third, and fourth moments of distribution are unremarkable (Figs. 5b, 5c, and 5d, respectively). On the other hand, the Lyapunov plot revealed the same abrupt changes in amplitude of the signal as the Sydney data in both the 1910s and in 1985 (Fig. 5e); this observation lends weight to the hypothesis that a transition also occurred in the 1910s in Melbourne. Finally, another abrupt change in the Lyapunov plot occurs in Melbourne in 1998 where the signal reverts to a larger amplitude. This latter transition heralds the onset of a significant acceleration in Melbourne's increasing mean maximum daily temperature, shown in the MMT plot (Fig. 5a).

Analysis of the maximum temperature record for Perth revealed an unsupervised optimal cluster size of 

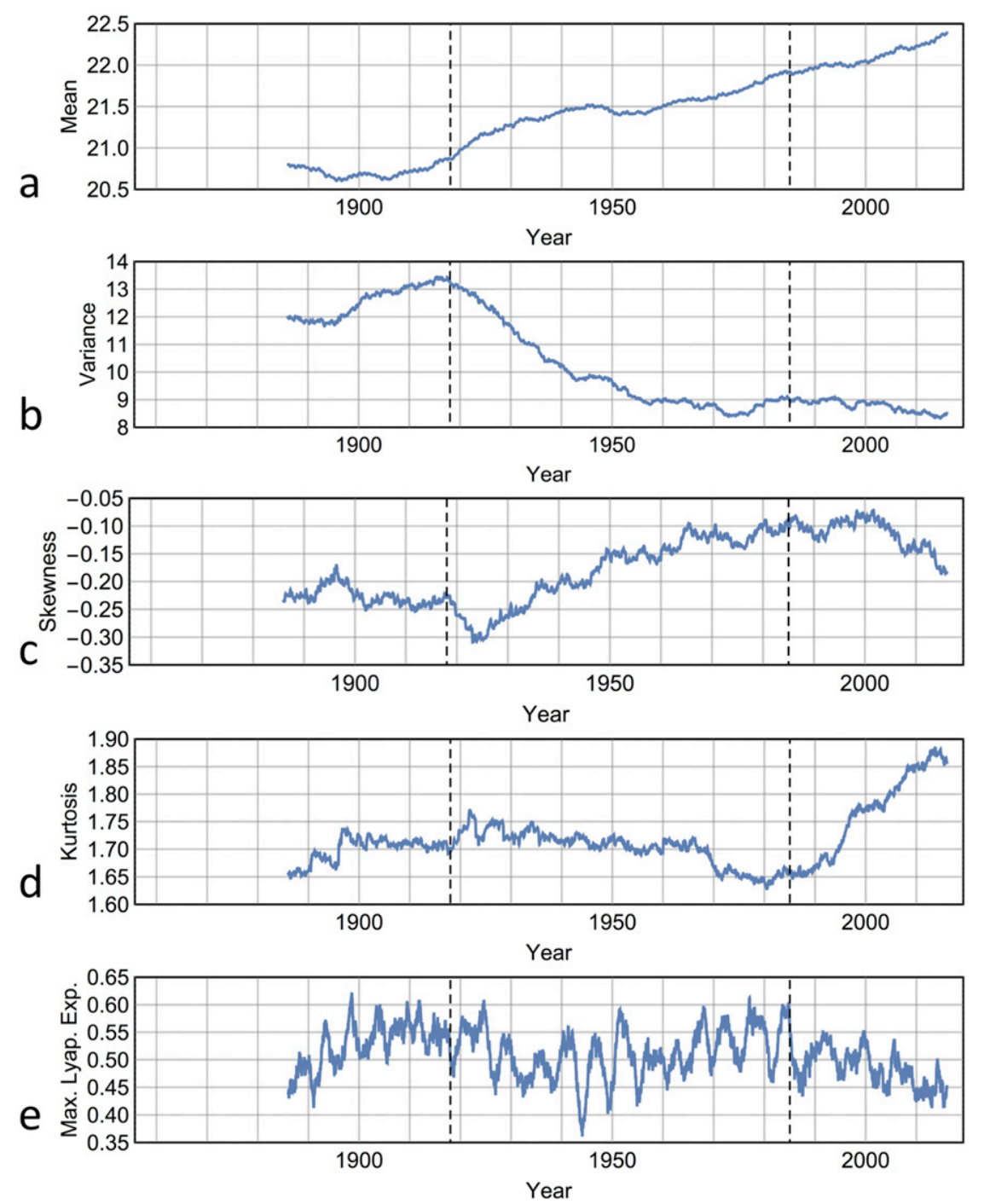

FIG. 4. Rolling window plots for Sydney with window length 1000 and lag 1: (a) MMT plot, (b) variance, (c) skewness, (d) kurtosis, and (e) Lyapunov exponents. The dashed lines correspond to the transition times suggested by the clustering analysis shown in Fig. 3. Note that the variance reaches a maximum at the first transition in 1918, and that the kurtosis increases to previously unseen levels after 1985. The Lyapunov plot reveals a pattern change in 1918 and in 1985, highlighted by the dashed lines.

two, a result validated by a majority-rule optimal cluster size among the 241 cluster-determining algorithms. Two-level FANNY clustering reveals a DPC of 0.832 , indicating the presence of well-defined clusters. Aggregation of the yearly cluster membership suggests that a transition occurred in the late 1980s or early 1990s (Fig. 6a); at this time the MMT plot reveals a mild acceleration in its increasing trend (Fig. 6c). The Lyapunov plot for Perth (Fig. 6e) revealed an abrupt change in the amplitude and pattern of the signal in 1991, consistent with the transition time as determined by the change in the slope in the MMT plot.
Analysis of these Hobart data revealed an unsupervised optimal cluster size of two; a result that was validated by a majority rule optimal cluster size with respect to the 241 cluster-determining algorithms. The aggregated yearly cluster membership reveals a boundary that occurred in the early 1970s (Fig. 6b), coincident with the onset of an increasing trend in the values of the MMT plot (Fig. 6d). Two-level FANNY clustering reveals a DPC of 0.778 , indicating the presence of reasonably well-defined clusters. The Lyapunov plot reveals a transition that also occurs in the early 1970s, corresponding to a shift from a largeamplitude to smaller-amplitude pattern (Fig. 6f). Note the 
a

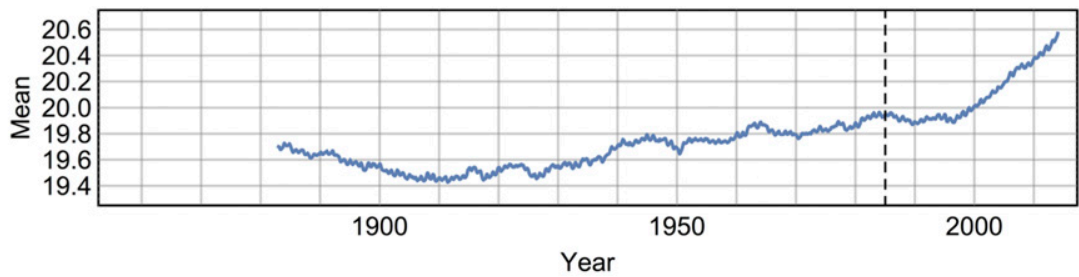

b
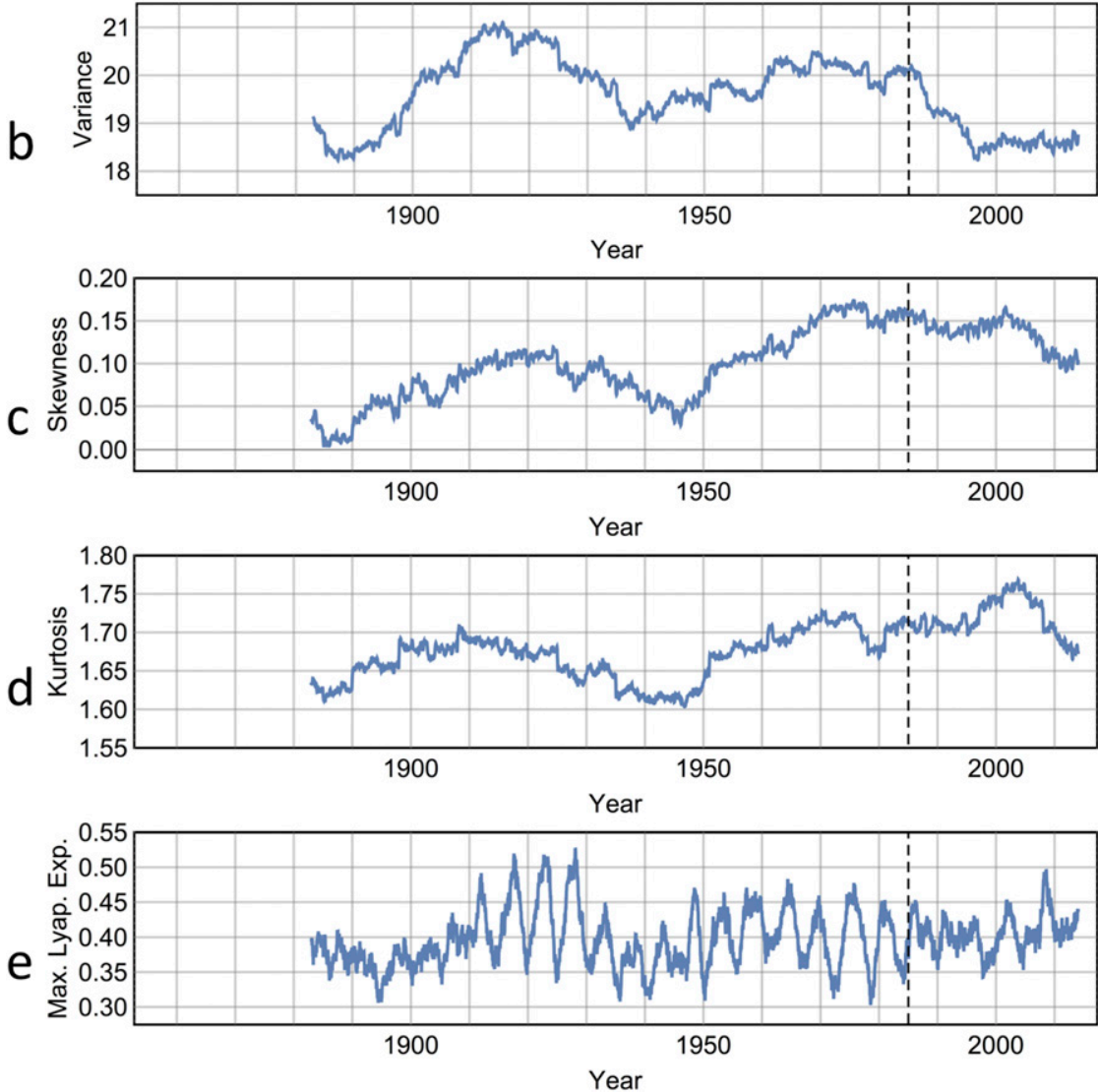

FIG. 5. Rolling window plots for Melbourne with window length 1000 and lag 1: (a) MMT plot, (b) variance, (c) skewness, (d) kurtosis, and (e) maximum Lyapunov exponents. The dashed line corresponds to the transition time suggested by the clustering analysis. Note that the mean rises sharply in 1998, corresponding to the time when the variance becomes constant. The Lyapunov signal shows a change in its pattern in 1985, corresponding to the transition suggested by the clustering analysis.

period between 1985 and 1992 where the amplitude of the signal is greatly diminished and is nonstationary.

Analysis of these Brisbane data revealed an unsupervised optimal cluster size of two, a result that was validated with the 241 cluster-determining algorithms. Temporal aggregation of the yearly cluster membership reveals a boundary in 1985 (Fig. 7a). Two-level FANNY clustering reveals a DPC of 0.816 , indicating the presence of well-defined clusters. The cluster boundary in 1985 is associated with the onset of an increasing trend in the values of the MMT plot (Fig. 7b). The Lyapunov plot, on the other hand, suggests that the transition occurred in 1980 . However, the early 1980s were associated with an exceptionally strong El Niño-in this case paradoxically associated with the presence of consecutive blue (cooler) bars in the clustering plot and a short-term decrease in the values of the MMT plot. Finally, note the change from a large to small-amplitude pattern in the Lyapunov plot around 2010.

The Adelaide data did not meaningfully partition into two or more clusters using the unsupervised clustering algorithms. The two-group supervised clustering did not reveal significant temporal aggregation (Fig. 7b). 

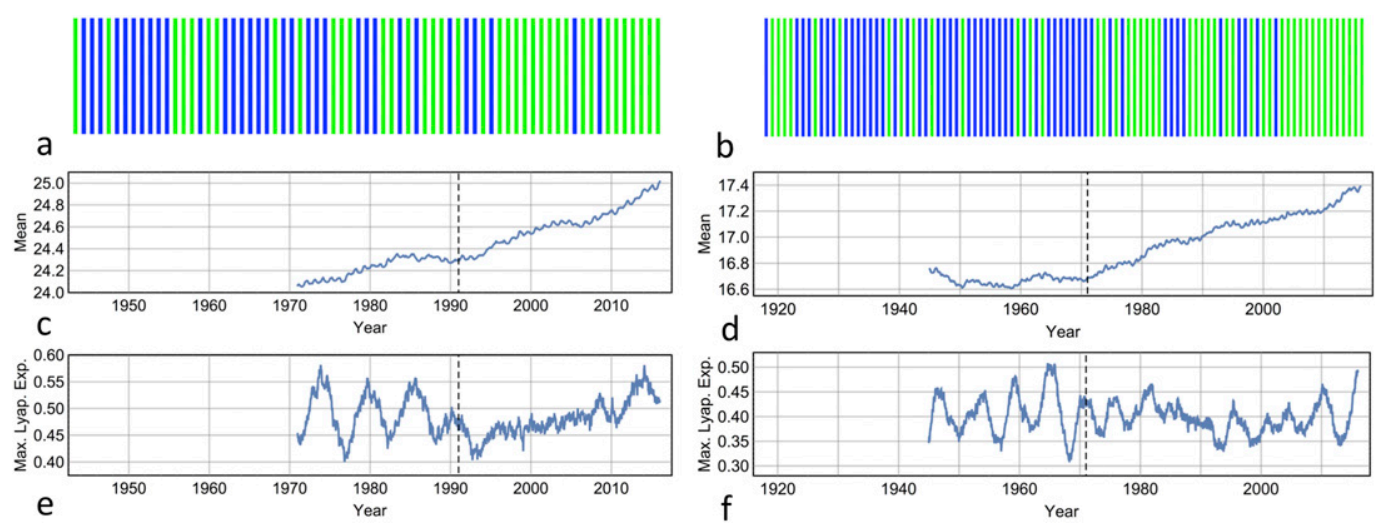

FIG. 6. Two-level FANNY clustering for (a) Perth and (b) Hobart. The MMT and Lyapunov plots for (c),(e) Perth and (d),(f) Hobart. The dashed line in (e) corresponds to the onset of increasing values in the MMT plot in (c), and a change in the pattern of the Lyapunov plot in (e). Likewise, the clustering transition in (b) is associated with increasing values of the MMT plot in (d), and the onset of a smaller-amplitude Lyapunov plot in (f).

Although a general warming trend is apparent, there is an absence of a well-defined boundary between temperature regimes. However, a significant increase in the slope of the MMT plot occurs in 2013; this corresponds to a change in the Lyapunov plot pattern from a large to a small-amplitude pattern (Fig. 7f).

Analysis of the maximum temperature record for Darwin did not reveal convincing evidence of clustering behavior. Unsupervised clustering revealed an optimal cluster size of 1, and 2-cluster supervised clustering demonstrated little temporal association with respect to the class membership. However, the Lyapunov plot revealed the typical large-amplitude pattern and a significant and abrupt reduction in the amplitude of the signal in 2009: this occurs within one year of the same change in the Brisbane Lyapunov plot (Fig. 7e).

\section{c. Power spectra}

Inspection of the Lyapunov plots derived from the Lorenz system and the temperature record suggest three broad categories of behavior:

(i) A stationary large-amplitude "sawtooth" pattern that exhibits minimal jaggedness (type I).

(ii) A stationary lower-amplitude pattern that exhibits a higher degree of jaggedness (type II).

(iii) A pattern where the large-scale amplitude almost vanishes; in these cases the evolution may be nonstationary (type III).
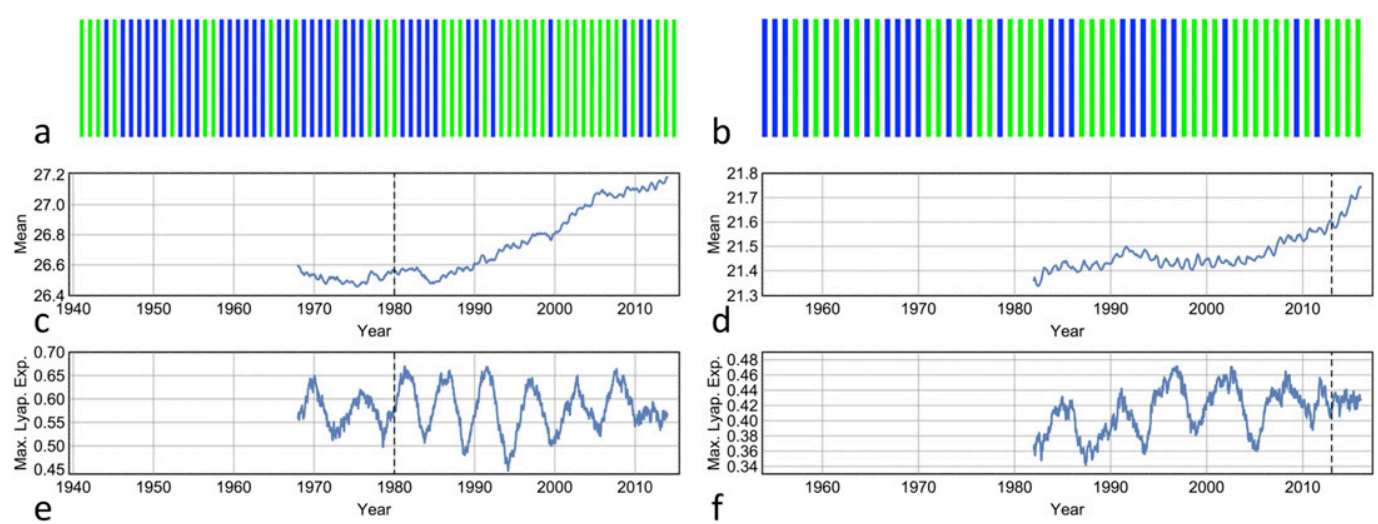

FIG. 7. Two-level FANNY clustering for (a) Brisbane and (b) Adelaide. The MMT and Lyapunov plots for (c),(e) Brisbane and (d),(f) Adelaide. The dashed line in (c) corresponds to a subtle change in the Lyapunov signal in (e), and is followed by a cooling period, shown as a cluster of blue bars and a dip in the MMT plot. At this time, paradoxically, a strong El Niño was active. Following this period, a strong warming trend ensued. Note that the clustering shown in (b) does not aggregate into well-defined groupings. Likewise, the Lyapunov plot does not reveal any change in its pattern until 2014, associated with an increase in the slope of the MMT plot in (b), but too recent for any clustering analysis to identify. 

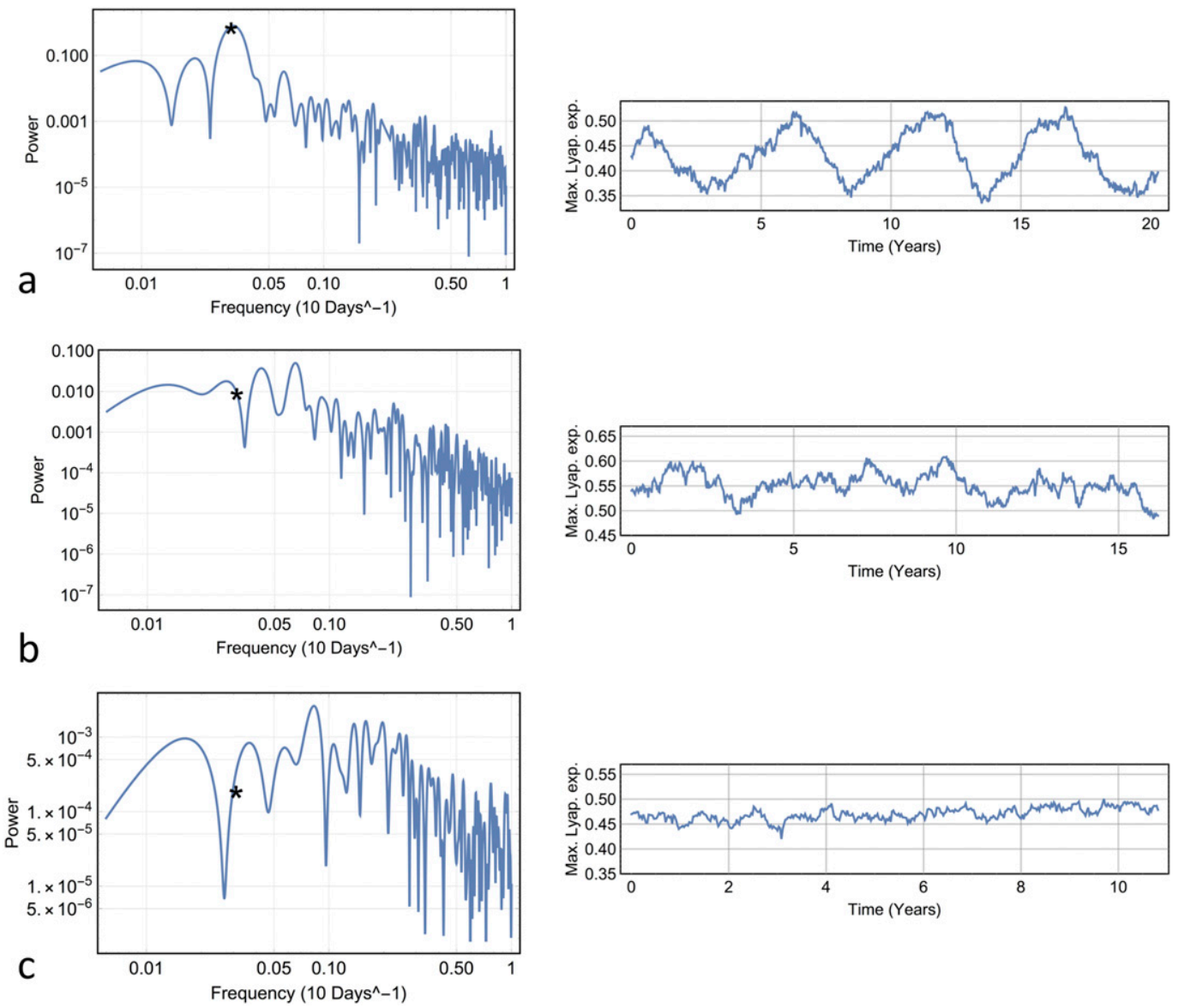

FIG. 8. Spectral analysis and associated Lyapunov plots of (a) type I, (b) type II, and (c) type III signals. Note that the amplitude of the Lyapunov signal diminishes significantly from type I to type III, and that the periodicity in the type I signal is not present in the type II or type III signals. The power falls as approximately $1 / f^{2}$ for the type I signal, approximating Brownian noise (see the text for details). Note that for the lower frequencies, the power is orders of magnitude greater in the type I signal. This is highlighted by the asterisk at $f=0.031$-here the power is given by $0.3,0.01$, and 0.0002 for the type I, type II, and type III signals, respectively. The units of $f$ are $(10 \text { days })^{-1}$ hence the corresponding period is 322 days.

Although visual inspection of the Lyapunov plots provides substantial evidence for transitions between type I, II, and III patterns-and thus provides evidence for the existence different modes with respect to system dynamics - analysis of the power spectra of the signals are likely to yield further insights. Figure 8 shows specific examples of the type I, II, and III patterns and their associated power spectra. Consider the type I pattern, present in all cities. Analysis of the power spectra for all cities revealed that the decay follows a power law given by $p \sim 1 / f^{\beta}$, where the exponent $\beta$ is the slope of the decay in the $\log -\log$ plot (Fig. 8). For the Melbourne, Sydney, Perth, Brisbane, Hobart, Adelaide, and Darwin spectra, the value of $\beta$ is given by $1.81,1.76,1.73,1.80,1.86,1.91$, and 1.89 , respectively. The patterns of fluctuations are thus tending toward Brownian noise, and are consistent Australia wide.

\section{d. The spectral power plot}

The major quantifiable difference in the power spectra between the types I, II, and III patterns as defined above is given by their respective power at a frequency within a narrow range near $f=0.031$, a finding that is independent of location (for specific examples, note the position of the three asterisks in the power spectra shown in Figs. 8a-c). For the type I pattern the mean power with respect to six cases (analysis of the Adelaide Lyapunov plot data were omitted here since the duration of its type I signal was short) at $f=0.031$ is given by 0.57 with a range of 0.33 to 1.03. In contrast, the power at $f=0.031$ with respect to the 3 cases of type II patterns revealed a mean power of 


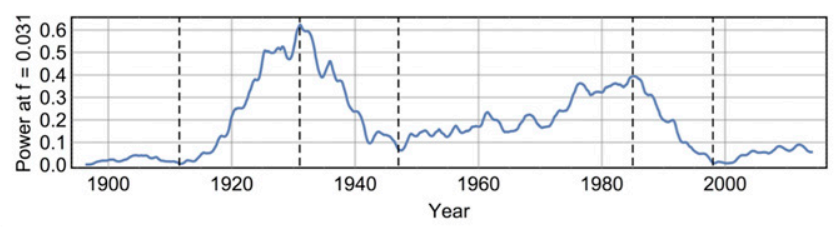

a
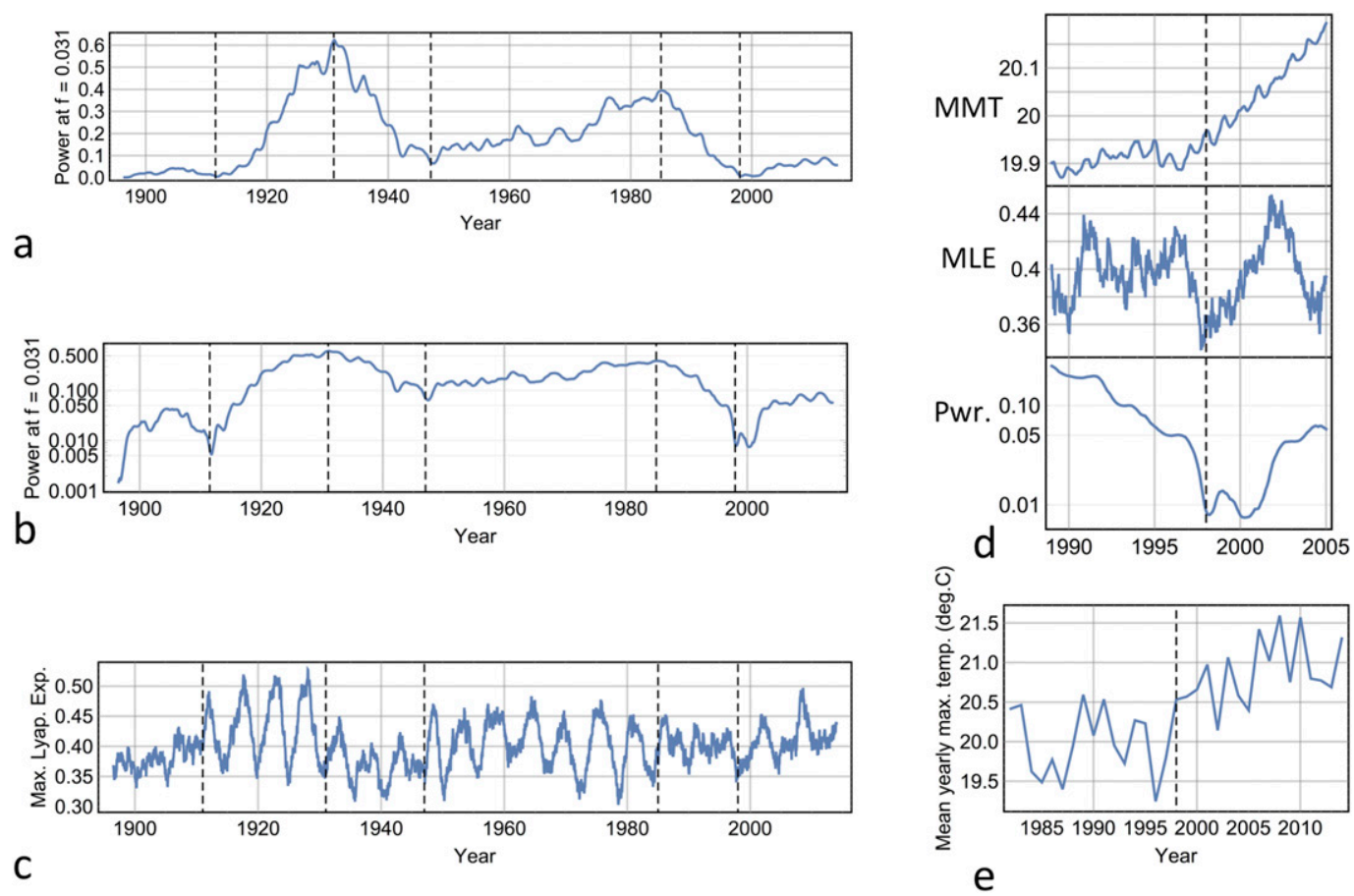

e

FIG. 9. (a) Melbourne's rolling window power plot, (b) the power plot with the $y$ axis scaled logarithmically, and (c) their associated Lyapunov plot. Note that the maxima and minima of the power plot associated with the reversal of long-term trends identify all the transitions in the patterns of the Lyapunov plot (dashed lines). The most recent transition in 1998 is shown in more detail in (d). This transition corresponds to a sharp upturn in the MMT plot. (e) Daily maximum temperatures averaged over 12 months taken from the original BOM data for 16 years either side of the 1998 transition. Note the discontinuity in the values of the state variable at the transition time, where a highly significant $1^{\circ} \mathrm{C}$ increase within 5 years is apparent.

0.0060 with a range of $0.0022-0.0088$. Finally, in the 3 cases of type III patterns the associated power at $f=$ 0.031 are an order of magnitude lower than the values associated with type II patterns, yielding a mean value of 0.00034 and a range of $0.00023-0.00044$. None of the other frequencies investigated were as discriminatory as $f=0.031$. Consider, for example, $f=0.10$. In this case the associated power of the type I, II, and III patterns are poor discriminators: their respective values are 0.001 , 0.001, and 0.0005 (Figs. 8a-c).

Although the sample sizes here are limited, these results suggest that the different types of signals present in the Lyapunov plots may be identifiable analytically, and thus may increase sensitivity with respect to the detection of different climate modes and their transitions. Figure 9a shows a rolling window spectral power plot for Melbourne at $f=0.031$ generated with a window length $N=500$ and $\operatorname{lag} L=1$ adjacent to its associated Lyapunov plot (Fig. 9c). Figure 9b shows the spectral power plot where the vertical axis is scaled logarithmically; this highlights the order-of-magnitude reductions in the power associated with plot minima. Note that the spectral power plot identifies additional transitions (cf. the dashed lines between Fig. 5e and Fig. 9c) as local maxima and minima associated with the reversal of longterm trends. Figures 9d and 9e show in greater detail the transition in Melbourne that occurred in 1998. Note that the minimum of the spectral power plot, the change in the pattern of the Lyapunov plot, and the onset in the upward trend of the MMT plot all occur simultaneously (see Table 1). Finally, the mean yearly maximum temperature with respect to the original BOM data for 16 years either side of this transition is shown in Fig. 9e. In this case it is clear that the mean value of the state variable has changed both discontinuously and significantly: it has increased by over $1^{\circ} \mathrm{C}$ within a few years.

Six further examples of climate transitions identified by Lyapunov plots and their associated spectral power plots are shown in Fig. 10. Note that the minima of the spectral power plots are associated with shifts in the Lyapunov plots from smaller to larger amplitudes, while the maxima are associated with shifts from larger to smaller amplitudes. All the plots in Fig. 10 reveal that the maxima or minima of the spectral power plots (which, like the Melbourne spectral power plots described above, are associated with the reversal of long-term 
Melbourne, 1911

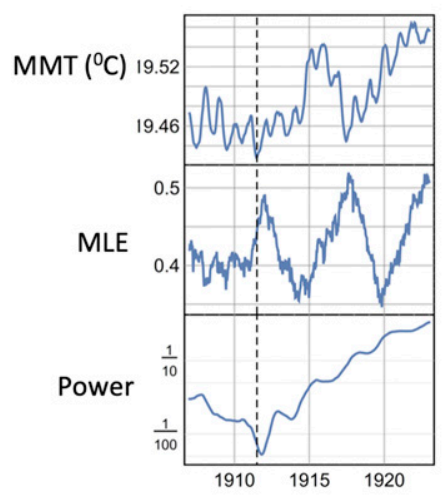

Hobart, 1969

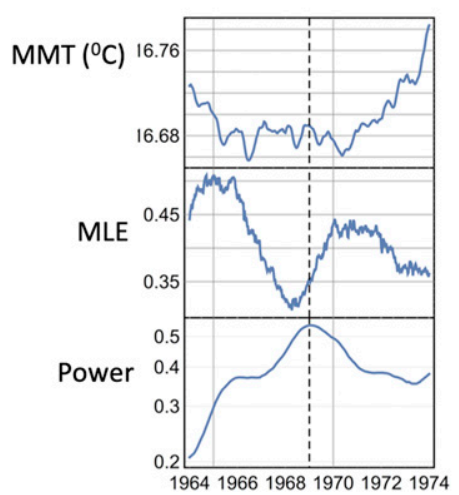

Sydney, 1918

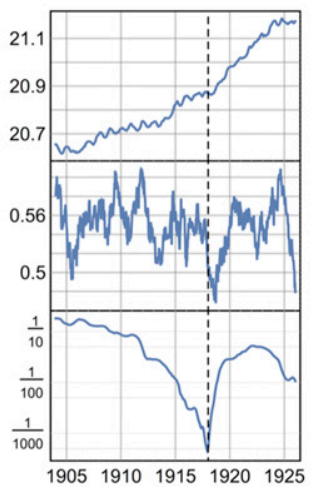

Perth, 2008

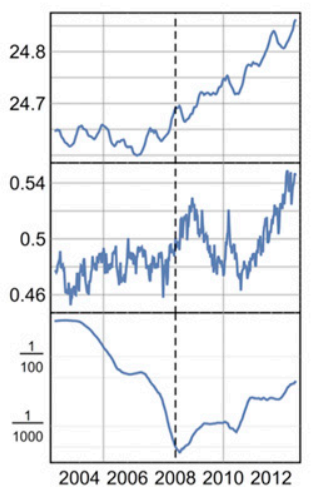

Sydney, 1956

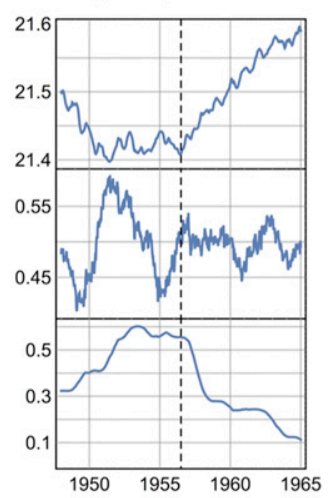

Hobart, 2009

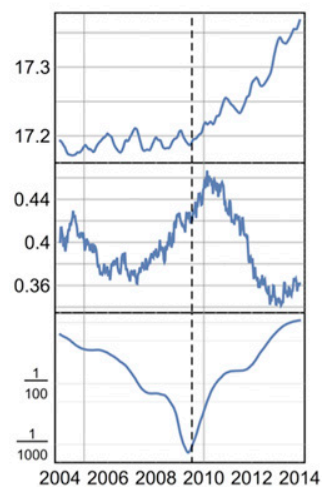

FIG. 10. Six examples of Australia-wide transitions associated with (i) the onset of a significant increase in the values of the MMT plot, (ii) a change in the pattern of the associated Lyapunov signal, and (iii) a local maxima or minima of the Lyapunov signal's rolling window power at $f=0.031$. Note that a large to small-amplitude change in the Lyapunov plot is associated with local maxima, while a small- to large-amplitude change is associated with local minima.

trends) occur at the same time as changes in the patterns of the Lyapunov plots, and all these changes herald the onset of accelerated upward trends in the values of the MMT plots.

The utility of the spectral power plot is illustrated by considering two examples: Perth in 2007 and Sydney in 1918. With respect to the former: is there another transition from the type III signal after 2005 (Fig. 6e)? Although visual inspection is suggestive, the spectral power plot is unequivocal: its values at $f=0.031$ reverse direction in 2008 and subsequently increase by two orders of magnitude, indicating a transition from a type III to a type I or type II pattern has occurred, heralding the onset of a significant increase in the slope of the MMT plot (Fig. 10). While the clustering analysis suggests a transition may have occurred in Sydney in 1918 (Fig. 3), the Lyapunov plot change is subtle and could be overlooked or dismissed. However, examination of the spectral power plot is again unequivocal: the rolling window power at $f=0.031$ decreases by two orders of magnitude immediately prior to the transition and then sharply recovers, revealing the presence of a significant change in the pattern of the Lyapunov signal (Fig. 10).

\section{e. Wavelet analysis}

Discrete and continuous time-frequency wavelet analysis of the Lyapunov signal was performed with respect to the transitions denoted by the dashed lines in Sydney (Fig. 4e), Melbourne (Fig. 5e, including the 1911 transition), Perth (Fig. 6e), Brisbane (Fig. 7e), and Hobart (Fig. 6f). The first six results are shown in Fig. 11, while the Hobart result is documented in Table 2. In all cases, significant changes in the values of the colorcoded coefficients of one or more frequency bands occur at the transition times; these events are striking in the continuous wavelet time-frequency analysis. 

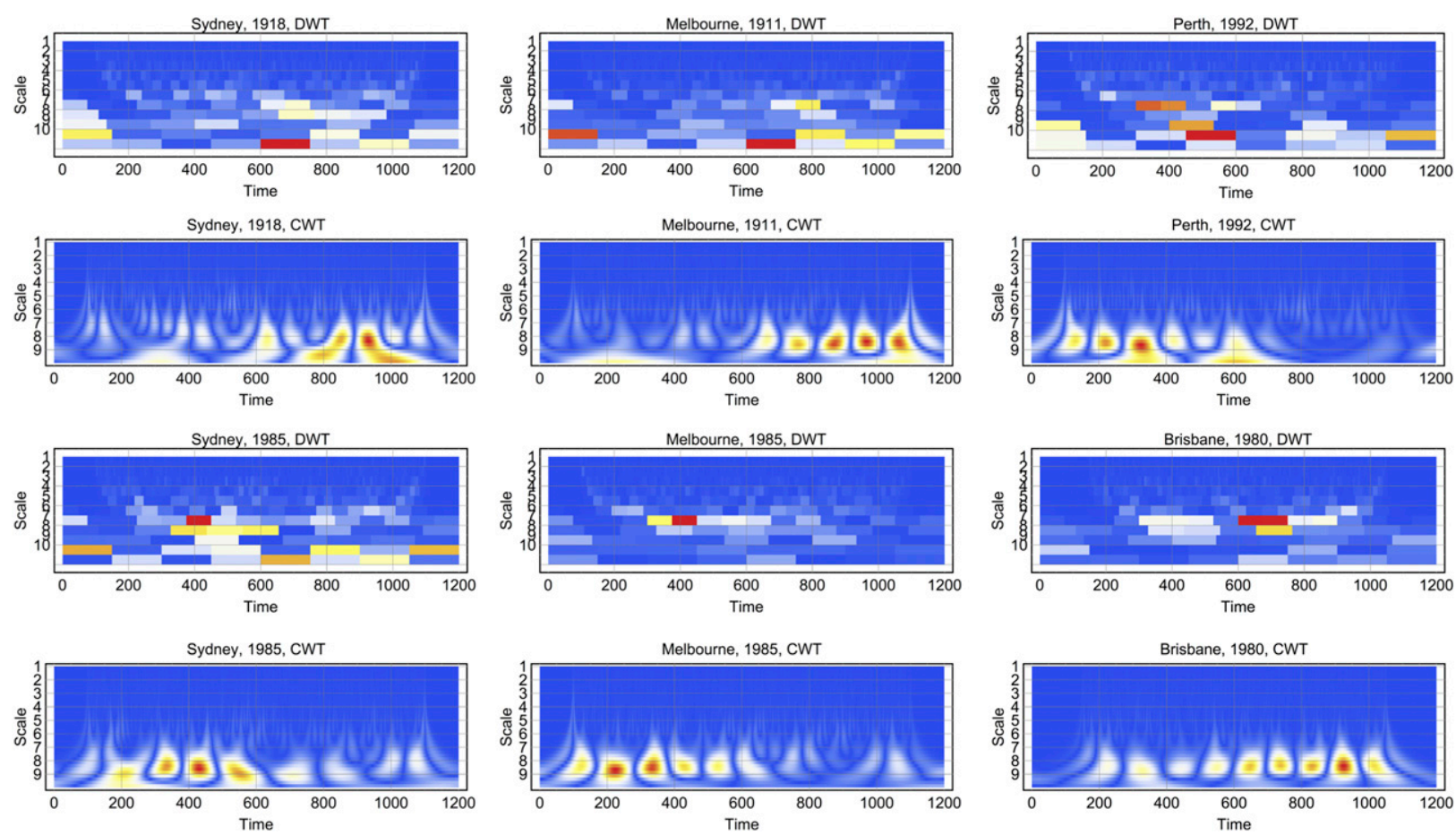

FIG. 11. Discrete (Daubechies 4) and continuous (second derivative Gaussian) time-frequency wavelet analysis (DWT and CWT) of the Lyapunov signal highlighting transitional behavior. The maps are organized from top to bottom then left to right corresponding to the following transitions: Sydney 1918 and 1985 (see dashed lines in Fig. 4e); Melbourne 1911 and 1985 (see dashed lines in Fig. 5e); Perth 1992 (see dashed line in Fig. 6e) and Brisbane 1980 (see dashed line in Fig. 7e). All transitions are translated such that they occur at the center of the maps at $t=600$. Note that abrupt changes occur over a range of frequencies at $t=600$; these are present in the DWT but more obvious in the CWT. Artifacts are present at $t<200$ and $t>1000$ because of boundary effects, hence the map accurately spans at least 400 data points, or approximately 12 years, either side of the transitions.

\section{f. Weather station metadata}

Examination of detailed metadata for all seven weather station locations did not reveal evidence for extraneous factors affecting the homogeneity of their respective temperature records. For example, consider instrument changes: the maximum temperature measurement instrumentation was installed in Sydney in 1859 and not removed until 1995; in Melbourne new instrumentation was installed in 1908 and replaced in 2001, 2002, and 2009; in Hobart instrumentation was installed in 1882 and replaced in 2000 and 2006. None of these years were identified as transitional years in the analysis presented. A mercury thermometer of type Dobbie was replaced with a WIKA mercury thermometer at the Perth weather station in 2009. Note from Fig. 10 that although a transition was identified from the Perth temperature record in 2008, it preceded the instrument change by one year. None of the other replacements in Perth $(2002,2015)$ were associated with any identified transitions. No instrument changes in Adelaide and Brisbane were temporally associated with any of the temperature transitions identified in this analysis.

\section{Discussion}

The results presented above, including the findings associated with generating Lyapunov plots from the Lorenz system, lend weight to the conclusion that multiple transitions have occurred with respect to Australian daily maximum temperatures within the last 100 years. However, from a practical perspective, it is the magnitude of the effect following the transition that is critical. It is possible to gauge the magnitude of the temperature shift by inspecting the change in slope of the MMT plots. While a sudden change in the magnitude of the derivative at the transition time adds to the weight of evidence favoring the existence of a transition, the increase in the magnitude of the derivative will be proportional to the magnitude of the shift. This follows from the observation that the rolling window mean includes data from the past 30 years; hence higher recent temperatures will increase the mean faster. Given the duration of the rolling window, the time taken for the MMT plot to reach equilibrium - if an equilibrium exists-will be 30 years, provided another transition does not occur. Since all the MMT plots reveal an increasing trend, it follows 
TABLE 2. Transition years for four Australian cities. In Sydney, for example, note that in 1918 all five measures change their behavior simultaneously, providing strong evidence for a chaos-to-chaos transition that defines distinct climate modes. In this case, the transition is identified by (i) a mean daily maximum temperature cluster boundary; (ii) an increase in the positive derivative of the MMT plot; (iii) a visual change in the Lyapunov signal pattern; (iv) a local minimum in the Lyapunov-associated spectral power plot; and (v) a change in the values of the coefficients associated with the discrete and continuous wavelet transform time-frequency maps (with respect to the Lyapunov signals). In all cities, the centroid coordinate values of the distinct clusters and the change in derivative of the MMT plots define new climate modes associated with warmer daily maximum temperature means.

\begin{tabular}{|c|c|c|c|c|c|}
\hline & Cluster boundary & $\begin{array}{l}\text { MMT plot positive increase } \\
\text { in derivative }\end{array}$ & $\begin{array}{l}\text { Lyapunov plot transition } \\
\text { (by inspection) }\end{array}$ & $\begin{array}{l}\text { Spectral power plot } \\
\text { maxima/minima }\end{array}$ & $\begin{array}{l}\text { Wavelet } \\
\text { identified }\end{array}$ \\
\hline \multirow[t]{3}{*}{ Sydney } & 1918 & 1918 & 1918 & 1918 & 1918 \\
\hline & 1985 & 1956 & 1956 & 1956 & 1985 \\
\hline & & & 1985 & & \\
\hline \multirow[t]{4}{*}{ Melbourne } & & 1911 & 1911 & 1911 & 1911 \\
\hline & 1985 & 1932 & 1932 & 1932 & 1985 \\
\hline & & 1998 & 1985 & 1985 & \\
\hline & & & 1998 & 1998 & \\
\hline \multirow[t]{2}{*}{ Hobart } & 1971 & 1969 & 1969 & 1969 & 1970 \\
\hline & & 2009 & 2009 & 2009 & \\
\hline \multirow[t]{2}{*}{ Perth } & 1993 & 1992 & 1991 & 1991 & 1992 \\
\hline & & 2008 & 2008 & 2008 & \\
\hline
\end{tabular}

that the 30-yr running average is out of equilibrium, consistent with the notion that the average time scale between transitions is not significantly greater than 30 years.

The largest temperature shift in Australia over the last 160 years (as determined from the available data) occurred in Melbourne in 1998 following an abrupt change in the amplitude of the Lyapunov plot (Fig. 9d). This event was associated with a rapid increase in the yearly average daily maximum temperature (Fig. 9e). Similar increases in the positive derivative of the MMT plot associated with abrupt changes in the pattern of the Lyapunov signal have also occurred in all other capital cities over the past 100 years (see Fig. 10). Since the MMT plot is subject to fluctuations and given that abrupt changes in the Lyapunov plot persist, it follows that the most sensitive method for quickly detecting a transition is by analyzing the Lyapunov signal. The findings reported here suggest that following both the Lyapunov plot and its associated spectral power plot at $f=0.031$ are likely to have utility in the early detection (but not prediction) of different Australian climate modes. The physical interpretation of the significance of this particular frequency, however, remains uncertain. In the case of a clean and simpler system such as the Lorenz system an abrupt switch to a new dynamical regime will become apparent almost immediately by simply following the Lyapunov plot pattern.

An interesting finding regarding the Lyapunov plots are the three signal patterns. While the type II signal is associated with a greater degree of chaos in the Lorenz system, it is unknown whether this association will apply to the temperature record. However, it is possible that the type III signal found in the analysis of the temperature record-in an analogous manner to the Lorenz system-represents a transient; here the Lyapunov exponents may be derived from a time series that span a rolling window before and after a transition. Such a pattern exists, for example, for a short period between two type I signals in the Hobart Lyapunov plot between 1983 and 1992 (Fig. 6f). This pattern may thus represent Lyapunov exponents derived from a time series mapped from two different attractors. By analogy with the second Lyapunov transition derived from the time series of the Lorenz system, the change in the Lyapunov signal in Hobart in 1992 may not then represent a dynamical system transition: the change to a type I signal in this case may indicate that the exponents are now derived from a time series mapped from a single attractor. In general, these considerations pose a problem for transition determination: how can a true transition be distinguished from a reversion to a unimodal signal? Although a definitive set of criteria will require further investigation, a true transition to a different dynamical regime is more likely to have occurred if (see Table 2)

(i) the Lyapunov transition occurs simultaneously in widely separated geographic locations [e.g., Sydney and Melbourne in 1985 (Figs. 4e, 5e)];

(ii) the Lyapunov signal transitions from a type I or type II pattern to a type III pattern;

(iii) the transition is associated with a state variable cluster transition (which can only be defined retrospectively; e.g., Sydney in 1918), or is associated with a change in the derivative of the MMT plot (e.g., Melbourne in 1998); 
(iv) the transition is associated with a local maxima or minima of the rolling window power (with respect to its Lyapunov signal) at $f=0.031$; and

(v) the transition is associated with an abrupt change in the values of the coefficients associated with one or more frequency bands with respect to the timefrequency discrete or continuous wavelet map.

The observation that the maximum Lyapunov exponents remain positive for all Lyapunov plots at all times implies that all the original maximum temperature time series are derived from chaotic systems. Although this observation implies that the transitions may represent chaos-to-chaos transitions, it is not possible from the analysis presented to determine precisely what the nature of an identified transition is-for example, whether it is a parameter-dependent boundary, interior, or merging crisis. Some dynamical systems may exhibit more than one strange attractor simultaneously, while others may be nonergodic; hence it is possible a trajectory can shift to a new location in phase space following a perturbation. However, the observation that the state variable (daily maximum temperature) has monotonically increased over a time scale comparable to the time scale associated with significant increases in atmospheric greenhouse gas concentrations, and the observation-found in the Lorenz system-that the Lyapunov plot pattern is invariant to a trajectory existing on different lobes of the same attractor, give pause to the idea that state changes are solely driven by natural or random variation.

Yet natural climate cycles exert a significant influence over global temperatures, are associated with warmer or cooler periods, and may flip from one state to the other secondary to perturbation. Consider the ENSO: the two strongest Southern Hemisphere El Niño events in recent decades occurred in 1982/83 and in 1997/98. The former event was temporally associated with a significant change in the pattern of Hobart's Lyapunov plot, while the latter event was temporally associated with the significant change in the pattern of Melbourne's Lyapunov plot. These findings perhaps suggest that these significant ENSO extremes were responsible for the Lyapunov plot changes. Yet in both Hobart and Melbourne the resultant climate modes persisted unchanged until 1992 and until at least 2016, respectively, indicating that the ENSO cannot be responsible for their persistence. Two of the strongest La Niña's on record occurred in 1973/74 and 2011. The latter event was temporally associated with a significant change in the pattern of the Lyapunov plot in Brisbane (Fig. 7e). Once again, however, the Lyapunov patterns persisted long after the La Niña had abated. All other Lyapunov- determined transitions were not temporally related to any other ENSO extremes. It is thus clear that the period of the ENSO is too short to account for the longer persistence of many of the identified climate modes; moreover, the ENSO is not acting as a trigger event for the majority of the Lyapunov-determined transitions.

The Indian Ocean dipole (IOD), analogous to the ENSO, is associated with periodic shifts between warm and cool modes (Saji et al. 1999), and exerts a strong influence on Australia's climate, particularly rainfall patterns. A very strong positive IOD event occurred in 1997 and a very strong negative IOD event occurred in 2011. These events were associated with transitions in the Lyapunov signal in Melbourne and Brisbane, respectively. The latter event was associated with an exceptionally strong La Niña; both the negative IOD and the La Niña were responsible for severe Australian flooding. Despite these associations, the period of the IOD is too short to account for the persistence of the climate modes identified by the Lyapunov plot transitions, and none of the other Lyapunov-determined transitions identified were associated with significant IOD events.

The southern annular mode (SAM) has undergone a positive trend since 1940, exhibiting its highest average annual positive value in 1997-98 (Abram et al. 2014), coincident with the abrupt change in Melbourne's Lyapunov plot. These large average annual positive values have persisted beyond 2000, coincident with persistence of the warmer climate regime in Melbourne identified by the Lyapunov signal. These events may be causally related: positive SAMs drive the Southern Hemisphere storm tracks poleward, leading to warmer and drier conditions in southern Australia. On the other hand, the short-term positive to negative fluctuations of the SAM, which occur over weekly to monthly time scales, are too short to be related to any climate modes determined by the Lyapunov signal. Stratospheric ozone depletion over the Antarctic was at its maximum in the late 1990s, and ozone depletion is considered a key driver of positive SAMs (Thompson et al. 2011). It is interesting to speculate that the convergence of four events in the late 1990s - maximal ozone depletion, a maximal positive SAM, a strong El Niño, and an strong positive IODacted in concert in the setting of anthropogenic forcing to trigger a chaotic transition in southern Australia's climate, resulting in the changes in the Lyapunov plot and the observed significant and abrupt increase in Melbourne's average daily maximum temperature.

The interdecadal Pacific oscillation (IPO) is the fourth major periodic influence on Australia's climate, shifting between positive and negative phases every few decades (Salinger et al. 2001). The two largest negative extremes 
occurred in 1918 and in the early to mid-1950s, coincident with Lyapunov-plot-determined transitions in Sydney (Fig. 10). The largest positive extreme occurred in the mid-1980s, coincident with Lyapunov-plotdetermined transitions in Sydney and Melbourne. The IPO shifted from a warm to a cool phase in the late 1990s, a transition that is considered an important driver of the global warming hiatus after 2000 (Dai et al. 2015). This event is coincident with a significant change in Melbourne's Lyapunov plot, where-paradoxically, given that the relationship between surface air temperature in Australia is positively correlated with warm phases of the IPO (Dong and Dai 2015)—yearly mean daily maximum temperatures increased discontinuously (Fig. 9e). In contrast to the ENSO, the IOD, and the SAM, the IPO has a decadal time scale, and its persistence in the cool phase after 2000 is coincident with the persistence of Melbourne's recent Lyapunov plot-determined climate regime. Over the last 100 years, the transition times between alternating positive and negative phases of the IPO $(1912,1924,1944,1978,1999)$ (Dong and Dai 2015) are suggestive of a coupling with the alternate climate mode boundaries determined by the Lyapunov signal in Melbourne (1911, 1932, 1946, 1985, 1998), (Fig. 9c) but the correspondence between years is not precise.

Although the global warming event that occurred in the 1920 s is unlikely to be related to anthropogenic forcing (Bengtsson et al. 2004), the impacts of both natural cycles and anthropogenic forcing are now known to play key roles in late twentieth-century warming (IPCC 2007). In northern Eurasia both atmospheric circulation effects and thermodynamic forcing are considered equally important in driving changes between climatic modes with different temperature regimes (Anisimov et al. 2013). It is possible that the interactions between the natural climate cycles' internal variability and greenhouse gas forcing-for example, resulting in an increased frequency of extreme El Niño events (Cai et al. 2014) —are triggering some of the Lyapunov-identified warming transitions and maintaining the subsequent climate modes. Anthropogenic forcing may have thus contributed to the extreme El Niño that occurred in 1998, triggering a Lyapunov plot-determined transition that was associated with a discontinuous and significant increase in Melbourne's mean yearly maximum temperature (Fig. 9e).

\section{Conclusions}

From a theoretical perspective it is known that some chaos-to-chaos transitions may be subtle, while others may be dramatic (Thompson et al. 1994). This observation is borne out in the MMT plots previously described: the magnitude of the derivative change with respect to the identifiable transitions is variable. It is important to note that while a significant and rapid change in the value of the yearly average maximum daily temperature may be obvious-such as in Melbourne in 1998 (Fig. 9e) - the absence of a dramatic shift discernable by eye in the time series of the state variable does not exclude the possibly of a transition. Now consider the predictability of the transitions described above. Unsurprisingly, given the ubiquity of positive maximum Lyapunov exponents in the analysis presented, none of the rolling window statistical measures exhibited any utility in the reliable prediction of any of the transitions. Indeed, there is no comfort in this finding: the inherent unpredictability of chaos-to-chaos transitions is coupled to an uncertainty regarding the magnitude of the effect (Thompson et al. 1994). This latter observation is important: identifying transitional behavior in the past implies that transitions will occur in the future. The absence of catastrophic transitions in the past does not then rule out the possibility-the results of general circulation models and thermodynamic constraints notwithstanding-of severe, unprecedented, and discontinuous increases in average daily maximum temperatures occurring in Australia at any time within the next few decades.

\section{REFERENCES}

Abarbanel, H. D. I., R. Brown, J. J. Sidorowich, and L. S. Tsimring, 1993: The analysis of observed chaotic data in physical systems. Rev. Mod. Phys., 65, 1331-1392, https://doi.org/10.1103/ RevModPhys.65.1331.

Abram, N. J., R. Mulvaney, F. Vimeux, S. J. Phipps, J. Turner, and M. H. England, 2014: Evolution of the southern annular mode during the past millennium. Nat. Climate Change, 4, 564-569, https://doi.org/10.1038/nclimate2235.

Anisimov, O., V. Kokorev, and Y. Zhil'tsova, 2013: Temporal and spatial patterns of modern climatic warming: Case study of northern Eurasia. Climatic Change, 118, 871-883, https:// doi.org/10.1007/s10584-013-0697-4.

Bathiany, S., H. Dijkstra, M. Crucifix, V. Dakos, V. Brovkin, M. S. Williamson, T. M. Lenton, and M. Scheffer, 2016: Beyond bifurcation: Using complex models to understand and predict abrupt climate change. Dyn. Stat. Climate Syst., 1, 1-31, https:// doi.org/10.1093/climsys/dzw004.

Bengtsson, L., V. A. Semenov, and O. M. Johannessen, 2004: The early twentieth-century warming in the Arctic-A possible mechanism. J. Climate, 17, 4045-4057, https://doi.org/10.1175/ 1520-0442(2004)017<4045:TETWIT>2.0.CO;2.

Bryant, P., R. Brown, and H. D. I. Abarbanel, 1990: Lyapunov exponents from observed time series. Phys. Rev. Lett., 65, 1523-1526, https://doi.org/10.1103/PhysRevLett.65.1523.

Cai, W., and Coauthors, 2014: Increasing frequency of extreme El Niño events due to greenhouse warming. Nat. Climate Change, 4, 111-116, https://doi.org/10.1038/nclimate2100. 
Cai, Y., K. L. Judd, T. M. Lenton, T. S. Lontzek, and D. Narita, 2015: Environmental tipping points significantly affect the cost-benefit assessment of climate policies. Proc. Natl. Acad. Sci. USA, 112, 4606-4611, https://doi.org/10.1073/pnas.1503890112.

Charrad, M., N. Ghazzali, V. Boiteau, and A. Niknafs, 2014: NbClust: An $\mathrm{R}$ package for determining the relevant number of clusters in a data set. J. Stat. Software, 61, 1-36, https:// doi.org/10.18637/jss.v061.i06.

Constantine, W., and D. Percival, 2014: fractal: Fractal Time Series Modeling and Analysis, version 2.0-0. R package, http:// CRAN.R-project.org/package $=$ fractal.

Dai, A., J. C. Fyfe, S. Xie, and X. Dai, 2015: Decadal modulation of global surface temperature by internal climate variability. Nat. Climate Change, 5, 555-559, https://doi.org/10.1038/ nclimate2605.

Dakos, V., M. Scheffer, E. H. van Nes, V. Brovkin, V. Petoukhov, and H. Held, 2008: Slowing down as an early warning signal for abrupt climate change. Proc. Natl. Acad. Sci. USA, 105, 14 308-14 312, https://doi.org/10.1073/pnas.0802430105.

Dechert, W. D., and R. Gencay, 1992: Lyapunov exponents as a nonparametric diagnostic for stability analysis. J. Appl. Econ., 7, S41-S60, https://doi.org/10.1002/jae.3950070505.

Ditlevsen, P. D., and S. J. Johnsen, 2010: Tipping points: Early warning and wishful thinking. Geophys. Res. Lett., 37, L19703, https://doi.org/10.1029/2010GL044486.

Dong, B., and A. Dai, 2015: The influence of the interdecadal Pacific oscillation on temperature and precipitation over the globe. Climate Dyn., 45, 2667-2681, https://doi.org/10.1007/ s00382-015-2500-x.

Dunn, J. C., 1974: Well separated clusters and optimal fuzzy partitions. J. Cybern., 4, 95-104, https://doi.org/10.1080/01969727408546059.

Fernandez-Rodriguez, F., S. Sosvilla-Rivero, and J. Andrada-Felix, 2005: Testing chaotic dynamics via Lyapunov exponents. J. Appl. Econ., 20, 911-930, https://doi.org/10.1002/jae.805.

Gorelov, A. S., 1996: Attractor dimension of the two-layer baroclinic model. Dokl. Earth Sci., 345A, 1-7.

Huang, C., A. G. Barnett, X. Wang, and S. Tong, 2012: The impact of temperature on years of life lost in Brisbane, Australia. Nat. Climate Change, 2, 265-270, https://doi.org/10.1038/ nclimate1369.

Huke, J. P., 1993: Embedding nonlinear dynamical systems: A guide to Takens' theorem. University of Manchester, $30 \mathrm{pp}$., http://eprints.maths.manchester.ac.uk/175/1/embed.pdf.

Ilyin, A. A., and A. N. Filatov, 1988: Unique solvability of NavierStokes equations on a two-dimensional sphere. Dokl. Akad. Nauk SSSR, 301, 18-22.

IPCC, 2007: Climate Change 2007: The Physical Science Basis. Cambridge University Press, 996 pp.

Kantz, H., and T. Schreiber, 1997: Nonlinear Time Series Analysis. Cambridge University Press, $304 \mathrm{pp}$.

Kennel, M. B., R. Brown, and H. D. I. Abarbanel, 1992: Determining embedding dimension for phase-space reconstruction using a geometrical construction. Phys. Rev., 45A, 3403-3411, https://doi.org/10.1103/PhysRevA.45.3403.

Ketchen, D. J., and C. L. Shook, 1996: The application of cluster analysis in strategic management research: An analysis and critique. Strategic Manage. J., 17, 441-458, https://doi.org/10.1002/ (SICI)1097-0266(199606)17:6<441::AID-SMJ819>3.0.CO;2-G.

Kluiving, R., H. W. Capel, and R. A. Pasmanter, 1992: Phase transitions within the fully developed chaotic regime. Chaotic Dynamics: Theory and Practice, T. Bountis, Ed., Plenum Press, 129-138.

Lai, Q., A. Dakgul, C. Li, G. Xu, and U. Çavu, 2018: A new chaotic system with multiple attractors: Dynamic analysis, circuit re- alization and S-Box design. Entropy, 20, 12, https://doi.org/ 10.3390/e20010012.

Lewis, S. C., A. D. King, and D. M. Mitchell, 2017: Australia's unprecedented future temperature extremes under Paris limits to warming. Geophys. Res. Lett., 44, 9947-9956, https:// doi.org/10.1002/2017GL074612.

Lions, J. L., O. P. Manley, R. Temam, and S. Wang, 1997: Physical interpretation of the attractor dimension for the primitive atmospheric circulation. J. Atmos. Sci., 54, 1137-1143, https://doi.org/10.1175/15200469(1997)054<1137: PIOTAD $>2.0 . \mathrm{CO} ; 2$

Lontzek, T. S., Y. Cai, K. L. Judd, and T. M. Lenton, 2015: Stochastic integrated assessment of climate tipping points indicates the need for strict climate policy. Nat. Climate Change, 5, 441-444, https://doi.org/10.1038/nclimate2570.

Lorenz, E. N., 1963: Deterministic nonperiodic flow. J. Atmos. Sci., 20, 130-141, https://doi.org/10.1175/1520-0469(1963)020<0130: $\mathrm{DNF}>2.0 . \mathrm{CO} ; 2$.

Lü, J., and G. Chen, 2002: A new chaotic attractor coined. Int. J. Bifurcation Chaos, 12, 659-661, https://doi.org/10.1142/ S0218127402004620.

Lucarini, V., and T. Bódai, 2017: Edge states in the climate system: Exploring global instabilities and critical transitions. Nonlinearity, 30, R32, https://doi.org/10.1088/1361-6544/aa6b11.

Luo, X., M. Small, M.-F. Danca, and G. Chen, 2007: On a dynamical system with multiple chaotic attractors. Int. J. Bifurcation Chaos, 17, 3235-3251, https://doi.org/10.1142/ S0218127407018993.

Maechler, M., P. Rousseeuw, A. Struyf, M. Hubert, K. Hornik, 2014: cluster: Cluster Analysis Basics and Extensions, version 1.15.3. R package, www.R-project.org/.

Mihailovic, D. T., G. Mimic, and I. Arsenic, 2014: Climate predictions: The chaos and complexity in climate models. $A d v$ Meteor., 2014, 878249, https://doi.org/10.1155/2014/878249.

Oyelade, J., I. Isewon, F. Oladipupo, O. Aromolaran, E. Uwoghiren, F. Ameh, M. Achas, and E. Adebiyi, 2016: Clustering algorithms: Their application to gene expression data. Bioinform. Biol. Insights, 10, 237-253, https://doi.org/10.4137/BBI.S38316.

Palmer, T. N., 1993: A nonlinear dynamical perspective on climate change. Weather, 48, 314-326, https://doi.org/10.1002/ j.1477-8696.1993.tb05802.x.

Perkins-Kirkpatrick, S. E., and Coauthors, 2016: Natural hazards in Australia: Heatwaves. Climatic Change, 139, 101-114, https:// doi.org/10.1007/s10584-016-1650-0.

R Core Team, 2014: R: A language and environment for statistical computing. R Foundation for Statistical Computing, www.Rproject.org/.

Reynolds, A., G. Richards, B. de la Iglesia, and V. Rayward-Smith, 2006: Clustering rules: A comparison of partitioning and hierarchical clustering algorithms. J. Math. Modell. Algorithms, 5, 475-504, https://doi.org/10.1007/s10852-005-9022-1.

Saji, N. H., B. N. Goswami, P. N. Vinayachan, and T. Yamagata, 1999: A dipole mode in the tropical Indian Ocean. Nature, 401, 360-363, https://doi.org/10.1038/43854.

Salinger, M. J., J. A. Renwick, and A. B. Mullan, 2001: Interdecadal Pacific oscillation and South Pacific climate. Int. J. Climatol., 21, 1705-1721, https://doi.org/10.1002/joc.691.

Sharples, J. J., and Coauthors, 2016: Natural hazards in Australia: Extreme bushfire. Climatic Change, 139, 85-89, https://doi.org/ 10.1007/s10584-016-1811-1.

Strogatz, S. H., 1994: Nonlinear Dynamics and Chaos: With Applications to Physics, Biology, Chemistry and Engineering. 1st ed. Addison-Wesley, 498 pp. 
Takens, F., 1981: Detecting strange attractors in turbulence. Dynamical Systems and Turbulence, Warwick 1980, Lecture Notes in Mathematics, Vol. 898, Springer-Verlag, 366-381.

Tanaka, T., K. Aihara, and M. Taki, 1996: Lyapunov exponents of random time series. Phys. Rev., 54E, 2122-2124, https:// doi.org/10.1103/PhysRevE.54.2122.

Thompson, D. W. J., S. Solomon, P. J. Kushner, M. H. England, K. M. Grise, and D. J. Karoly, 2011: Signatures of the Antarctic ozone hole in Southern Hemisphere surface climate change. Nat. Geosci., 4, 741-749, https://doi.org/10.1038/ngeo1296.

Thompson, J. M. T., H. B. Stewart, and Y. Ueda, 1994: Safe, explosive and dangerous bifurcations in dissipative dynamical systems. Phys. Rev., 49E, 1019-1027, https://doi.org/10.1103/ PhysRevE.49.1019.

Tibshirani, R., G. Walther, and T. Hastie, 2001: Estimating the number of data clusters via the Gap statistic. J. Roy. Stat. Soc., 63B, 411-423, https://doi.org/10.1111/14679868.00293.
Tong, J.-L., H. Wu, W. Hou, W.-P. He, and J. Zhou, 2014: Early warning signals of abrupt temperature change in different regions of China over the past 50 years. Chin. Phys. B, 23, 049201, https://doi.org/10.1088/1674-1056/23/4/049201.

Torrence, C., and G. P. Compo, 1998: A practical guide to wavelet analysis. Bull. Amer. Meteor. Soc., 79, 61-78, https://doi.org/ 10.1175/1520-0477(1998)079<0061:APGTWA>2.0.CO;2.

Varotsos, C. A., C. L. E. Franzke, M. N. Efstathiou, and A. G. Degermendzhi, 2014: Evidence for two abrupt warming events of SST in the last century. Theor. Appl. Climatol., 116, 51-60, https://doi.org/10.1007/s00704-013-0935-8.

Williams, G. P., 1997: Chaos Theory Tamed. Joseph Henry Press, $520 \mathrm{pp}$.

Zheng, Z., J. Lu, G. Chen, T. Zhou, and S. Zhang, 2004: Generating two simultaneously chaotic attractors with a switching piecewise-linear controller. Chaos Solitons Fractals, 20, 277-288, https://doi.org/10.1016/S0960-0779(03) 00376-X. 